\title{
CONVERGENCE TO INFINITELY DIVISIBLE DISTRIBUTIONS WITH FINITE VARIANCE FOR SOME WEAKLY DEPENDENT SEQUENCES
}

\author{
JÉRÔME DEDECKER ${ }^{1}$ AND SANA LOUHICHI ${ }^{2}$
}

\begin{abstract}
We continue the investigation started in a previous paper, on weak convergence to infinitely divisible distributions with finite variance. In the present paper, we study this problem for some weakly dependent random variables, including in particular associated sequences. We obtain minimal conditions expressed in terms of individual random variables. As in the i.i.d. case, we describe the convergence to the Gaussian and the purely non-Gaussian parts of the infinitely divisible limit. We also discuss the rate of Poisson convergence and emphasize the special case of Bernoulli random variables. The proofs are mainly based on Lindeberg's method.
\end{abstract}

Mathematics Subject Classification. 60E07, 60F05.

Received July 3, 2003.

\section{INTRODUCTION}

Infinitely divisible distributions play a fundamental role in the area of limit theorems of probability theory. This role is described by the following result due to Khintchine: let $\left(X_{j, n}\right)_{1 \leq j \leq n}$ be a triangular array of i.i.d. random variables such that $X_{0, n}$ converges in probability to zero as $n$ tends to infinity. Then the set of distribution that are limits (in the sense of weak convergence) of the distribution of $\sum_{j=1}^{n} X_{j, n}$ coincides with the set of all infinitely divisible distribution (see for instance [25]).

A probability measure $\mu$ on $\mathbb{R}$ is infinitely divisible if, for any positive integer $n$, there is a probability measure $\mu_{n}$ on $\mathbb{R}$ such that $\mu=\mu_{n}^{n}$, where $\nu^{n}$ denotes the $n$-fold convolution of the probability measure $\nu$ with itself. Since 1930, we know that the the characteristic function $\hat{\mu}$ of any infinitely divisible distribution $\mu$ is given by the formula

$$
\hat{\mu}(z)=\exp \left(-\frac{1}{2} \sigma^{2} z^{2}+i \gamma z+\int\left(\mathrm{e}^{i z x}-1-i z x \mathbb{I}_{\{|x| \leq 1\}}(x)\right) \nu(\mathrm{d} x)\right), \quad \text { for any } z \in \mathbb{R}
$$

\footnotetext{
Keywords and phrases. Infinitely divisible distributions, Lévy processes, weak dependence, association, binary random variables, number of exceedances.

${ }^{1}$ Laboratoire de Statistique Théorique et Appliquée, Université Paris 6, Site Chevaleret, 13 rue Clisson, 75013 Paris, France; dedecker@ccr.jussieu.fr

2 Laboratoire de Probabilités, Statistique et modélisation, Université de Paris-Sud, Bât. 425, 91405 Orsay Cedex, France; Sana.Louhichi@math.u-psud.fr

(c) EDP Sciences, SMAI 2005
} 
where $\sigma, \gamma$ are real numbers and $\nu$ is a positive measure on $\mathbb{R}$ such that

$$
\nu(\{0\})=0 \text { and } \int\left(x^{2} \wedge 1\right) \nu(\mathrm{d} x)<+\infty .
$$

This representation of $\hat{\mu}$ by the triplet $\left(\sigma^{2}, \nu, \gamma\right)$ is unique. The formula (0.1) is known as the Lévy-Khintchine representation and $\left(\sigma^{2}, \nu, \gamma\right)$ as the generating triplet of $\mu$. The quantities $\sigma^{2}$ and $\nu$ are called, respectively, the Gaussian variance and the Lévy measure of $\mu$.

If $\int_{|x| \geq 1} x^{2} \nu(\mathrm{d} x)$ is finite, then so is $\int x^{2} \mu(\mathrm{d} x)$. If moreover $\int x \mu(\mathrm{d} x)=0$, then we have the representation by the couple $\left(\sigma^{2}, \nu\right)$

$$
\hat{\mu}(z)=\exp \left(-\frac{1}{2} \sigma^{2} z^{2}+\int\left(\mathrm{e}^{i z x}-1-i z x\right) \nu(\mathrm{d} x)\right), \quad \text { for any } z \in \mathbb{R},
$$

which is equivalent to

$$
\hat{\mu}(z)=\exp \left(\int\left(\mathrm{e}^{i z x}-1-i z x\right) \frac{1}{x^{2}} \mathrm{~d} F(x)\right), \quad \text { for any } z \in \mathbb{R},
$$

where $F$ is the distribution function of some finite measure (i.e. $F$ is bounded, nondecreasing, $F(-\infty)=0$ and $F(\infty)$ is finite). The distribution function $F$ is called Kolmogorov's spectral function. Let $\mu_{F}^{1}$ denotes the probability measure with characteristic function given by (0.3) and define for any positive real $t$ the probability $\mu_{F}^{t}=\mu_{t F}^{1}$. The distribution $\mu_{F}^{t}$ has mean zero and variance $t F(\infty)$ and satisfies the equation $\mu_{F}^{t} * \mu_{F}^{s}=\mu_{F}^{t+s}$.

The connection between $F$ and the generating couple $\left(\sigma^{2}, \nu\right)$ is given by

$$
F(x)=\int_{-\infty}^{x} t^{2} \nu(\mathrm{d} t)+\sigma^{2} \mathbb{1}_{[0,+\infty[}(x) .
$$

Clearly $F(\infty)=\int t^{2} \nu(\mathrm{d} t)+\sigma^{2}=\int x^{2} \mu(\mathrm{d} x)$. The Gaussian variance $\sigma^{2}$ is related to $F$ via the relation

$$
\lim _{\epsilon \rightarrow 0}(F(\epsilon)-F(-\epsilon))=\sigma^{2}
$$

Hence the continuity of $F$ at zero ensures that $\sigma^{2}=0$. In such a case $\mu$ is called purely non-Gaussian. Examples of centered infinitely divisible distributions $\mu$ with finite second moment are then given by the function $F$ or equivalently by the generating couple $\left(\sigma^{2}, \nu\right)$. The Gaussian measure is obtained when the Lévy measure $\nu$ is zero i.e. when $F=\sigma^{2} \mathbb{1}_{[0,+\infty[}$. The infinitely divisible distribution $\mu$ is Poisson if $\sigma^{2}=0$, and $\nu=\lambda \delta_{1}$ or equivalently when $F=\lambda \mathbb{I}_{[1,+\infty[}$. More generally, the Coumpound Poisson measure is obtained when $\sigma^{2}=0$ and $\nu=\lambda \rho$, where $\rho$ is a probability measure on $\mathbb{R}$ such that $\rho(\{0\})=0$. In that case $F(x)=\lambda \int_{\infty}^{x} t^{2} \rho(\mathrm{d} t)$. Other examples of infinitely divisible distributions on $\mathbb{R}$ can be found in [28].

Let $\left(X_{j, n}\right)_{1 \leq j \leq n}$ be a triangular array of stationary centered random variables such that $\mathbb{E}\left(X_{0, n}^{2}\right)$ converges to zero as $n$ tends to infinity. Suppose that for each $n,\left(X_{i, n}\right)_{1 \leq i \leq n}$ are i.i.d. From Theorem 2 of Chapter 4 in [12], we know that $S_{n}(t)=X_{1, n}+\cdots+X_{[n t], n}$ converges in distribution to $\mu_{F}^{t}$ if and only if, for any continuity point $x$ of $F$,

$$
\lim _{n \rightarrow \infty} n \mathbb{E}\left(X_{0, n}^{2} \mathbb{I}_{X_{0, n} \leq x}\right)=F(x) .
$$

In this paper, our aim is to extend the result of Gnedenko and Kolmogorov to certain dependent sequences. When it is relevant, we also study the convergence of the Donsker line $\left\{S_{n}(t), t \in[0,1]\right\}$ to a Lévy process in the space of cadlag functions equipped with Skorohod's distance. A stochastic process $\left\{X_{t}, t \geq 0\right\}$ on $\mathbb{R}$ is a Lévy process if the following conditions are satisfied.

(1) For any choice of $n \geq 1$ and $0 \leq t_{0}<t_{1}<\ldots<t_{n}$, the random variables $X_{t_{0}}, X_{t_{1}}-X_{t_{0}}, X_{t_{2}}-X_{t_{1}}$, $\ldots, X_{t_{n}}-X_{t_{n-1}}$ are independent. 
(2) $X_{0}=0$ a.s

(3) The distribution of $X_{t+s}-X_{s}$ does not depend on $s$.

(4) It is stochastically continuous.

(5) Almost surely, $X_{t}(\omega)$ is right-continuous in $t \geq 0$ and has left limits in $t>0$.

The only Lévy process with almost sure continuous paths is the Brownian motion while the only Lévy process with almost sure count paths is the Poisson process (a count path is a nondecreasing cadlag function which takes integers as values and has jumps of exactly 1 at its point of discontinuity).

Our paper is organized as follows. In Section 1 we study the problem of convergence to infinitely divisible distributions for sequences having an independent asymptotic representation ( $c f$. Condition (1.1)). Under this condition convergence in distribution to $\mu_{F}$ is equivalent to

$$
\lim _{p \rightarrow \infty} \limsup _{n \rightarrow+\infty}\left|p \mathbb{E}\left(S_{n}^{2}(1 / p) \mathbb{I}_{S_{n}(1 / p) \leq x}\right)-F(x)\right|=0
$$

(cf. Th. 1, Sect. 1). To prove this result we adapt Lindeberg's method with increasing blocks in place of individual variables. The main idea is to split $S_{n}(1)$ into $p$ blocks distributed as $S_{n}(1 / p)$ and to replace them step by step by blocks of i.i.d. variables with law $\mu_{F}^{1 / n}$.

Once the convergence of $S_{n}(1)$ to $\mu_{F}$ is guaranteed, it remains to describe the sequences constructed from the original one, that approximate the Gaussian and the purely non-Gaussian parts of the infinitely divisible law $\mu_{F}$. For this, we consider the truncated random variables

$$
X_{i, n}(\eta)=f_{\eta}\left(X_{i, n}\right)-\mathbb{E}\left(f_{\eta}\left(X_{i, n}\right)\right) \quad \text { and } \quad X_{i, n}^{(\eta)}=f^{(\eta)}\left(X_{i, n}\right)-\mathbb{E}\left(f^{(\eta)}\left(X_{i, n}\right)\right),
$$

where the "decoupling" functions $f_{\eta}$ and $f^{(\eta)}$ are defined by $f_{\eta}(x)=(\eta \wedge x) \vee(-\eta)$ and $f^{(\eta)}(x)=x-f_{\eta}(x)$. In Propositions 1 and 2 we prove that, under a "Lindeberg-type condition" ( $c f$. Condition $\left(\mathcal{C}_{1}\right)$ of Prop. 1), the small sum (i.e. the sum of the random variables which are small by truncation) is approximately normal with variance asymptotically equal to the jump of $F$ at 0 . While the essential sum (i.e. the sum of the essential parts of the variables) is approximated by a purely non-Gaussien distribution, provided that the quantity

$$
p \mathbb{E}\left(\left(\sum_{i=1}^{[n / p]} X_{i, n}^{(\eta)}\right)^{2} \mathbb{1}_{\left|\sum_{i=1}^{[n / p]} X_{i, n}^{(\eta)}\right| \leq \epsilon}\right)
$$

is sufficiently small ( $c f$. Condition $\left(\mathcal{C}_{3}\right)$ of Prop. 1). The total sum behaves as a convolution of the normal and the purely non-Gaussian distribution ( $c f$. Prop. 2). For i.i.d. sequences this result is a consequence of the so-called "découpage de Lévy" method (see for instance Lemma 3-1 in [1]). The key for the proofs of Propositions 1 and 2 is Lemma 4 which reduces the behaviour of the total sum to that of the essential sum, as soon as the small variables obey a "Lindeberg-type condition".

In Section 2, we apply the results of Section 1 to associated random variables. This notion of positive dependence comes independently from physics (mostly through the FKG inequality, cf. [11]), reliability and statistics [3,10]. In Theorem 2 we give sufficient conditions on the individual random variables (or at least on some finite sums of the random variables) for the finite dimensional distributions of $S_{n}(t)$ to converge to those of a Lévy process. More precisely the main conditions are: the sequences $\operatorname{Var} S_{n}(1)$ and $\operatorname{Var} S_{n}^{(\eta)}(1)$ converge respectively to some positive constants $F(\infty)$ and $G(\infty)$, and for any positive integer $N$

$$
\lim _{\eta \rightarrow 0} \limsup _{n \rightarrow+\infty} n\left[\mathbb{E}\left(S_{N, n}^{(\eta) 2} \mathbb{I}_{S_{N, n}^{(\eta)} \leq x}\right)-\mathbb{E}\left(S_{N-1, n}^{(\eta) 2} \mathbb{I}_{S_{N-1, n}^{(\eta)} \leq x}\right)\right]=F_{N}(x)
$$

where $F_{N}$ is a bounded variation (BV) function. If so $F_{N}$ converges weakly to the distribution function $G$ of some finite measure. The sum $S_{n}(1)$ is then approximated by the convolution of the normal law with variance 
$F(\infty)-G(\infty)$ and $\mu_{G}$ (see Th. 2 for more details). In Section 2.2, we study the convergence to Lévy processes (cf. Th. 3 and Prop. 3). We then deduce sufficient conditions for the convergence to Wiener or Poisson processes ( $c f$. Cors. 3 and 4). In Section 2.3, we consider the special case of Bernoulli distributed random variables. Let $\left(Y_{i, n}\right)$ be an array of associated and Bernoulli distributed random variables with parameter $p_{n}$ such that $n p_{n}$ is bounded. We suppose that the variance of the corresponding sum $S_{n, n}(Y)$ converges and that (0.4) holds for $S_{N, n}(Y)$. We prove that the limiting law is integer-valued coumpound poisson. When $\operatorname{Cov}\left(X_{0, n}, X_{i, n}\right)=0$ for $i \geq m+1$, the conditions that we impose are comparable to the necessary and sufficient conditions obtained by [15] for $m$-dependent Bernoulli-distributed arrays ( $c f$. Cor. 5 and the remarks below for more details). Finally, we give a bound for the Dudley distance between a sum of Bernoulli random variables and the Poisson distribution by using Lindeberg's method once more (see the proof of Prop. 4). Those results apply to stationary and Gaussian sequences with positive correlation function and provide upper bounds for the number of exceedances close to that obtained by [13].

In Section 3, we give the proofs for weakly dependent sequences. In particular, we give useful bounds for Conditions $\left(\mathcal{C}_{1}\right)$ and $\left(\mathcal{C}_{3}\right)$ (cf. Lems. 5 and 6 respectively). In Section 4, we give the proofs for associated sequences. The rates for the convergence to the Poisson distribution are proved in Section 5 (cf. Th. 4).

\section{WEAK CONVERGENCE TO INFINITELY DiVISIBLE DISTRIBUtion FOR SOME DEPENDENT SEQUENCES}

In this section we give an analogue of Theorem 1 in [8] for some sequences satisfying other type of dependence. More precisely, our dependence assumptions are comparable to Condition B in [16] and [17] (cf. Condition (1.1) below). This condition suggests an asymptotic independent representation of the sum $S_{n}(1)$ by blocs of independent random variables: there exists an i.i.d. sequence $\left(\tilde{U}_{i, n}\right)$ with marginal distribution $S_{n}\left(1 / p_{n}\right)$, such that the sum $\tilde{S}_{n}=\sum_{i=1}^{p_{n}} \tilde{U}_{i, n}$ and $S_{n}(1)$ have the same limiting behaviour under suitable conditions on $p_{n}$.

Theorem 1. Let $\left(X_{i, n}\right)_{i \in \mathbb{N}, n \in \mathbb{N}}$ be a stationary array of square integrable and centered real-valued random variables and define $S_{n}(t)=X_{1, n}+\cdots+X_{[n t], n}$. Suppose that $\mathbb{E}\left(X_{0, n}^{2}\right)$ tends to zero as $n$ tends to infinity. Assume that, for any $z \in \mathbb{R}$ and any $t \in[0,1]$,

$$
\lim _{p \rightarrow+\infty} \limsup _{n \rightarrow+\infty}\left|\mathbb{E} \exp \left(i z S_{n}(t)\right)-\left(\mathbb{E} \exp \left(i z S_{[n t]}(1 / p)\right)\right)^{p}\right|=0
$$

Let $F$ be the distribution function of some finite measure. The following statements are equivalent:

(i) For any $t \in[0,1]$, the sequence $\left(S_{n}(t)\right)_{n \geq 0}$ converges in distribution to the probability $\mu_{F}^{t}$.

(ii) For any continuity point $x$ (including $+\infty$ ) of $F$,

$$
\lim _{p \rightarrow \infty} \limsup _{n \rightarrow+\infty}\left|p \mathbb{E}\left(S_{n}^{2}(1 / p) \mathbb{I}_{S_{n}(1 / p) \leq x}\right)-F(x)\right|=0 .
$$

Note that the nature of Condition (1.2) is very close to that of condition LD in [17]. In both cases, the idea is to calculate the parameters of the limiting distribution on the base of properties of distributions of finite sums.

Remark. Let $S_{m, n}=X_{1, n}+\cdots+X_{m, n}$ and define the set $\mathcal{E}$ of all positive integers $m$ such that

$$
\forall z \in \mathbb{R}, \quad \forall t \in[0,1], \quad \lim _{n \rightarrow+\infty}\left|\mathbb{E} \exp \left(i z S_{n}(t)\right)-\left(\mathbb{E} \exp \left(i z S_{m, n}\right)\right)^{[[n t] / m]}\right|=0 .
$$

Let $m_{0}(X)$ be an element of the set $\mathcal{E}$, with the convention that $m_{0}(X)=+\infty$ if $\mathcal{E}=\emptyset$. The term $\lim _{m \rightarrow m_{0}(X)} f(m)$ will designate either $f\left(m_{0}(X)\right)$ if $m_{0}(X)$ is finite, or the usual limit if $m_{0}(X)=\infty$. Instead of (1.1), we can suppose that the sum $S_{n}(t)$ admits an asymptotic independent representation by a sum of i.i.d. blocs with length $m$ independent of $n$ :

$$
\lim _{m \rightarrow m_{0}(X)} \limsup _{n \rightarrow+\infty}\left|\mathbb{E} \exp \left(i z S_{n}(t)\right)-\left(\mathbb{E} \exp \left(i z S_{m, n}\right)\right)^{[[n t] / m]}\right|=0
$$


If we assume moreover, instead of (1.2) that there exists a distribution function $F$ such that for any continuity point $x$ (including $+\infty$ ) of $F$,

$$
\lim _{m \rightarrow m_{0}(X)} \limsup _{n \rightarrow+\infty}\left|\frac{n}{m} \mathbb{E}\left(S_{m, n}^{2} \mathbb{I}_{S_{m, n} \leq x}\right)-F(x)\right|=0
$$

then the conclusion of Theorem 1 still holds. An interesting case arises when 1 belongs to $\mathcal{E}$. In such a case the sequence behaves as its independent version, as shown in the following corollary.

Corollary 1. Let $\left(X_{i, n}\right)$ be as in Theorem 1. Suppose that for any $z \in \mathbb{R}$, and any $t \in[0,1]$

$$
\lim _{n \rightarrow+\infty}\left|\mathbb{E} \exp \left(i z S_{n}(t)\right)-\left(\mathbb{E} \exp \left(i z X_{0, n}\right)\right)^{[n t]}\right|=0 .
$$

Let $F$ be a given distribution function. Then the statement (i) of Theorem 1 is equivalent to the following: For any continuity point $x$ (including $+\infty$ ) of $F$,

$$
\lim _{n \rightarrow+\infty} n \mathbb{E}\left(X_{0, n}^{2} \mathbb{I}_{X_{0, n} \leq x}\right)=F(x) .
$$

We shall justify this remark in the proof of Theorem 1.

We now discuss an equivalent statement for (1.2). For $\eta>0$, consider the two 1-Lipschitz functions $f_{\eta}$ and $f^{(\eta)}$ defined by $f_{\eta}(x)=(\eta \wedge x) \vee(-\eta)$ and $f^{(\eta)}(x)=x-f_{\eta}(x)$. Set

$$
X_{i, n}(\eta)=f_{\eta}\left(X_{i, n}\right)-\mathbb{E}\left(f_{\eta}\left(X_{i, n}\right)\right) \quad \text { and } \quad X_{i, n}^{(\eta)}=X_{i, n}-X_{i, n}(\eta)
$$

and define for any $t \in[0,1]$

$$
S_{n}^{(\eta)}(t)=\sum_{i=1}^{[n t]} X_{i, n}^{(\eta)} \quad \text { and } \quad S_{n, \eta}(t)=\sum_{i=1}^{[n t]} X_{i, n}(\eta)
$$

Clearly $S_{n}(t)=S_{n}^{(\eta)}(t)+S_{n, \eta}(t)$. For sufficiently small $\eta$, the random variable $X_{i, n}(\eta)$ represents small values, while $X_{i, n}^{(\eta)}$ is the essential part of $X_{i, n}$. Under a "Lindeberg-type condition" on the small sum $S_{n, \eta}(t)$, the behaviour of the original sum $S_{n}(t)$ is described by its essential part $S_{n}^{(\eta)}(t)$ as shown in the following proposition.

Proposition 1. Let $\left(X_{i, n}\right)$ be a stationary array of square integrable and centered real-valued random variables. Consider the three conditions

$\left(\mathcal{C}_{1}\right)$ For any $\epsilon>0 \quad \lim _{\eta \rightarrow 0} \limsup _{p \rightarrow+\infty} \limsup _{n \rightarrow+\infty} p \mathbb{E}\left(S_{n, \eta}^{2}(1 / p) \mathbb{I}_{\left|S_{n, \eta}(1 / p)\right| \geq \epsilon}\right)=0$.

$\left(\mathcal{C}_{2}\right)$ The four sequences $\left(n \mathbb{E} X_{0, n}^{2}\right),\left(V_{n}(p)\right)=\left(p \operatorname{Var} S_{n}(1 / p)\right),\left(V_{n, \eta}(p)\right)=\left(p \operatorname{Var} S_{n, \eta}(1 / p)\right)$ and $\left(V_{n}^{(\eta)}(p)\right)=$ $\left(p \operatorname{Var} S_{n}^{(\eta)}(1 / p)\right)$ are uniformly bounded over $n, p$ and $\eta$.

$\left(\mathcal{C}_{3}\right) \lim _{\epsilon \rightarrow 0} \limsup _{\eta \rightarrow 0} \limsup _{p \rightarrow+\infty} \limsup _{n \rightarrow+\infty} p \mathbb{E}\left(S_{n}^{(\eta) 2}(1 / p) \mathbb{I}_{-\epsilon<S_{n}^{(\eta)}(1 / p) \leq \epsilon}\right)=0$.

If $\mathcal{C}_{1}, \mathcal{C}_{2}$ and $\mathcal{C}_{3}$ hold, then (1.2) is equivalent to: for any continuity point $x$ of $F$

$$
\lim _{\eta \rightarrow 0} \limsup _{p \rightarrow+\infty} \limsup _{n \rightarrow+\infty}\left|p \mathbb{E}\left(S_{n}^{(\eta) 2}(1 / p) \mathbb{I}_{S_{n}^{(\eta)}(1 / p) \leq x}\right)-F(x)+\mathbb{I}_{x \geq 0}\left(V_{n}(p)-V_{n}^{(\eta)}(p)\right)\right|=0 .
$$

In particular, letting $\sigma^{2}=\lim _{\epsilon \rightarrow 0}(F(\epsilon)-F(-\epsilon))$, then $\left(\mathcal{C}_{1}\right),\left(\mathcal{C}_{2}\right),\left(\mathcal{C}_{3}\right)$ and (1.2) ensure that

$$
\lim _{\eta \rightarrow 0} \limsup _{p \rightarrow+\infty} \limsup _{n \rightarrow+\infty}\left|V_{n}(p)-V_{n}^{(\eta)}(p)-\sigma^{2}\right|=0 .
$$


In Sections 3.4 and 3.5 we give useful inequalities in order to check Conditions $\left(\mathcal{C}_{1}\right)$ and $\left(\mathcal{C}_{3}\right)$. We suppose now that Conditions $(1.1),\left(\mathcal{C}_{1}\right),\left(\mathcal{C}_{2}\right),\left(\mathcal{C}_{3}\right)$ and $(1.2)$ are all satisfied. Our main task in the following proposition is to describe the sequences constructed from $\left(X_{i, n}\right)$ that approximate respectively the Gaussian part and the purely non-Gaussian part. Let $\left(\tilde{U}_{i, n}\right),\left(\tilde{U}_{i, n}(\eta)\right),\left(\tilde{U}_{i, n}^{(\eta)}\right)$ be three sequences of i.i.d. r.v's distributed as $S_{n}(1 / p)$, $S_{n, \eta}(1 / p)$ and $S_{n}^{(\eta)}(1 / p)$, respectively. Define

$$
\tilde{S}_{n}:=\sum_{i=1}^{p} \tilde{U}_{i, n}, \quad \tilde{S}_{n}^{(\eta)}:=\sum_{i=1}^{p} \tilde{U}_{i, n}^{(\eta)}, \quad \tilde{S}_{n, \eta}:=\sum_{i=1}^{p} \tilde{U}_{i, n}(\eta)
$$

The following proposition shows that for small $\eta$, the distribution $\mathcal{L}\left(S_{n}(1)\right)$ of the partial sum $S_{n}(1)$ is well approximated by the convolution of $\mathcal{L}\left(\tilde{S}_{n}^{(\eta)}\right)$ and $\mathcal{L}\left(\tilde{S}_{n, \eta}\right)$. The two distributions $\mathcal{L}\left(\tilde{S}_{n}^{(\eta)}\right)$ and $\mathcal{L}\left(\tilde{S}_{n, \eta}\right)$ are respectively approximated by a purely non-Gaussian distribution and a Gaussian distribution.

Proposition 2. Let $\left(X_{i, n}\right)_{i \in \mathbb{N}, n \in \mathbb{N}}$ be as in Theorem 1 and define the functions $G_{n, p}^{(\eta)}$ by the equality $G_{n, p}^{(\eta)}(x)=$ $F(x)-\mathbb{I}_{x \geq 0}\left(V_{n}(p)-V_{n}^{(\eta)}(p)\right)$. If $(1.1),\left(\mathcal{C}_{1}\right),\left(\mathcal{C}_{2}\right)$ and $\left(\mathcal{C}_{3}\right)$ are all satisfied, then the statement $(i)$ of Theorem 1 (with $t=1$ ) is equivalent to:

$$
\begin{aligned}
& \lim _{\eta \rightarrow 0} \limsup _{p \rightarrow+\infty} \limsup _{n \rightarrow+\infty}\left|\mathbb{E}\left(\exp \left(i z \tilde{S}_{n, \eta}\right)\right)-\exp \left(-\frac{z^{2}}{2} V_{n, \eta}(p)\right)\right|=0 . \\
& \lim _{\eta \rightarrow 0} \limsup _{p \rightarrow+\infty} \limsup _{n \rightarrow+\infty}\left|\mathbb{E}\left(\exp \left(i z \tilde{S}_{n}^{(\eta)}\right)\right)-\exp \left(\int \frac{\mathrm{e}^{i z x}-1-i z x}{x^{2}} \mathrm{~d} G_{n, p}^{(\eta)}(x)\right)\right|=0 . \\
& \lim _{\eta \rightarrow 0} \limsup _{p \rightarrow+\infty} \limsup _{n \rightarrow+\infty} \operatorname{Cov}\left(S_{n}^{(\eta)}(1 / p), S_{n, \eta}(1 / p)\right)=0 . \\
& \lim _{\eta \rightarrow 0} \limsup _{p \rightarrow+\infty} \limsup _{n \rightarrow+\infty} \mid \mathbb{E}\left(\exp \left(i z S_{n}\right)-\mathbb{E}\left(\exp \left(i z \tilde{S}_{n, \eta}\right)\right) \mathbb{E}\left(\exp \left(i z \tilde{S}_{n}^{(\eta)}\right)\right) \mid=0 .\right.
\end{aligned}
$$

Remark. Let $G(x)=F(x)-\mathbb{1}_{x \geq 0}\left(F\left(0^{+}\right)-F\left(0^{-}\right)\right)$. This function inherits all the properties of $F$ and is continuous at 0 . To summarize, Conditions $(1.1),\left(\mathcal{C}_{1}\right),\left(\mathcal{C}_{2}\right),\left(\mathcal{C}_{3}\right)$ and (i) of Theorem 1 prove that the distribution of $\left(\tilde{S}_{n}^{(\eta)}\right)$ is approximated by the purely non-Gaussian Lévy distribution $\mu_{G}$ while the distribution of $\left(\tilde{S}_{n, \eta}\right)$ is described by the Gaussian distribution with variance $F\left(0^{+}\right)-F\left(0^{-}\right)$(also equals to $F(+\infty)-G(+\infty)$ ).

\section{Convergence to LÉvy processes For Associated VARIABles}

We now apply the results of the previous section to stationary arrays fulfilling a condition of positive dependence called association. We also discuss convergence to Lévy processes for such sequences.

Let $\left(X_{i, n}\right)_{i \in \mathbb{N}, n \in \mathbb{N}}$ be as in Theorem 1. It is an array of associated random variables if for every $n$ :

$$
\operatorname{Cov}\left(h\left(X_{i, n}, i \in A\right), k\left(X_{i, n}, i \in B\right)\right) \geq 0,
$$

where $h$ and $k$ are coordinatewise nondecreasing real-valued functions and $A, B$ are finite subsets of $\mathbb{N}$ (we refer to $[3,10]$ and the references therein for more details on associated random variables).

Up to our knowledge, the convergence to infinitely divisible distributions for arrays of associated variables is studied separately in the case of Gaussian or Poisson distributions ( $c f$. [22] for an overview). For a stationary sequence $\left(X_{i}\right)$ of square integrable random variables, the Gaussian limits are obtained as soon as the Cox-Grimmett coefficient ( $c f .[7])$

$$
U(n):=2 \sum_{j=n+1}^{+\infty} \operatorname{Cov}\left(X_{1}, X_{j}\right)
$$


tends to zero as $n$ tends to infinity. Poisson limits, or more generally convergence results for triangular arrays of independent variables, are extended to associated variables provided that

$$
\lim _{n \rightarrow+\infty} \sum_{1 \leq j<k \leq n} \operatorname{Cov}\left(X_{j, n} X_{k, n}\right)=0
$$

(cf. Prop. 1 in [23]). In order to get a more general result containing both Gaussian and Poisson limits, we generalize the Cox-Grimmett coefficient: let

$$
R_{a}(N)=\limsup _{n \rightarrow+\infty} n \sum_{r=N}^{n} \operatorname{Cov}\left(X_{0, n}, X_{r, n}\right) .
$$

Let $N_{a, 0}(X)=\inf \left\{N, R_{a}(N)=0\right\}\left(N_{a, 0}(X)\right.$ may be infinite). The main assumptions are

$$
\begin{aligned}
& \lim _{N \rightarrow N_{a, 0}(X)} \limsup _{n \rightarrow+\infty} n \sum_{r=N}^{n} \operatorname{Cov}\left(X_{0, n}, X_{r, n}\right)=0 \text { and } \\
& \lim _{N \rightarrow N_{a, 0}(X)} \limsup _{n \rightarrow+\infty}\left|n \mathbb{E}\left(X_{0, n}^{2}\right)+2 n \sum_{r=1}^{N-1} \operatorname{Cov}\left(X_{0, n}, X_{r, n}\right)-F(\infty)\right|=0,
\end{aligned}
$$

where $F(\infty)$ is some nonnegative number. For stationary arrays of associated variables, Condition (2.2) with $N_{a, 0}(X)=1$ is exactly $(2.1)$.

\subsection{Convergence of the finite dimensional distributions}

We introduce the analogue of Condition $B(N)$ defined in [8].

$B^{\prime}(N)$ : There exists a BV function $F_{N}$ such that for any continuity point $x$ of $F_{N}$,

$$
\lim _{\eta \rightarrow 0} \limsup _{n \rightarrow+\infty} n\left[\mathbb{E}\left(S_{N, n}^{(\eta) 2} \mathbb{I}_{S_{N, n}^{(\eta)} \leq x}\right)-\mathbb{E}\left(S_{N-1, n}^{(\eta) 2} \mathbb{I}_{S_{N-1, n}^{(\eta)} \leq x}\right)\right]=F_{N}(x) .
$$

As for $\left(X_{i, n}\right)$, define

$$
R^{(a)}(N)=\limsup _{\eta \rightarrow 0} \limsup _{n \rightarrow+\infty} n \sum_{r=N}^{n} \operatorname{Cov}\left(X_{0, n}^{(\eta)}, X_{r, n}^{(\eta)}\right) .
$$

and $N_{0}^{(a)}(X)=\inf \left\{N, R^{(a)}(N)=0\right\}$. We make the same assumptions on $\left(X_{i, n}^{(\eta)}\right)_{i}$ as we make on $\left(X_{i, n}\right)$, that is

$$
\begin{aligned}
& \lim _{N \rightarrow N_{0}^{(a)}(X)} \limsup _{\eta \rightarrow 0} \limsup _{n \rightarrow+\infty} n \sum_{r=N}^{n} \operatorname{Cov}\left(X_{0, n}^{(\eta)}, X_{r, n}^{(\eta)}\right)=0 \text { and } \\
& \lim _{N \rightarrow N_{0}^{(a)}(X)} \limsup _{\eta \rightarrow 0} \limsup _{n \rightarrow+\infty}\left|n \mathbb{E}\left(X_{0, n}^{(\eta) 2}\right)+2 n \sum_{r=1}^{N-1} \operatorname{Cov}\left(X_{0, n}^{(\eta)}, X_{r, n}^{(\eta)}\right)-G(\infty)\right|=0,
\end{aligned}
$$

where $G(\infty)$ is some positive real number.

Theorem 2. Let $\left(X_{i, n}\right)_{i \in \mathbb{N}, n \in \mathbb{N}}$ be a stationary array of associated square integrable and centered real-valued random variables. Suppose that $\mathbb{E}\left(X_{0, n}^{2}\right)$ tends to zero as $n$ tends to infinity. Assume that Conditions (2.2), (2.3), (2.5) and (2.6) hold. Assume moreover that there exists a nondecreasing sequence of positive integers $\left(N_{i}\right)_{i \in \mathbb{N}^{*}}$ converging to $N_{0}^{(a)}(X)$ such that $B^{\prime}\left(N_{i}\right)$ holds for any $i$ in $\mathbb{N}^{*}$. Then there exists a distribution function $G$ such that, for any bounded and three times continuously differentiable function $g$ with compactly supported derivatives

$$
\lim _{N_{i} \rightarrow N_{0}^{(a)}} \int g \mathrm{~d} F_{N_{i}}=\int g \mathrm{~d} G .
$$


If moreover $G$ is continuous at 0 , then the finite dimensional distributions of the process $\left\{S_{n}(t), t \in[0,1]\right\}$ converges in distribution to those of the Lévy process of law $\mu_{F}$, where $F$ is given by

$$
F(x)=G(x)+\mathbb{I}_{0<x}(F(\infty)-G(\infty)) .
$$

Recall that $F(\infty)$ and $G(\infty)$ have been defined in (2.3) and (2.6).

Remark. Suppose that, for each fixed $N$, the BV function $F_{N}$, given in $B^{\prime}(N)$ is of the form $F_{N}(x)=$ $\sum_{j=1}^{N} \lambda_{j, N} \mathbb{I}_{j<x}$, where $\left(\lambda_{j, N}\right)$ is a sequence of real numbers. Suppose moreover that the requirements of Theorem 2 are satisfied. Then the distribution function $G$, whose existence is guaranteed by Theorem 2 satisfies $G(x)=$ $\sum_{j=1}^{N_{0}^{(a)}(X)} \lambda_{j} \mathbb{1}_{j<x}$, where the positive numbers $\lambda_{j}$ are the limit of $\lambda_{j, N}$. In this case the limit law is given by its characteristic function

$$
\hat{\mu}(z)=\exp \left(-(F(\infty)-G(\infty)) \frac{z^{2}}{2}+\sum_{j=1}^{N_{0}^{(a)}(X)} \lambda_{j}\left(\mathrm{e}^{i j z}-1-i j z\right) \frac{1}{j^{2}}\right) .
$$

This law is the convolution of some Gaussian distribution with some integer-valued Coumpound Poisson distribution.

\subsection{Convergence to Lévy processes}

Let $D([0,1])$ be the space of cadlag functions equipped with Skorohod's distance $d$. Convergence in the Skorohod topology is somewhat restrictive: for instance if $x_{n}=\mathbb{1}_{[1 / 2,1]}$ and $y_{n}=\mathbb{I}_{[1 / 2-1 / n, 1]}$, both sequences converge in $(D([0,1]), d)$ but $x_{n}+y_{n}$ is not even relatively compact in that space. It means that for the convergence in $(D([0,1], d))$, two jumps of given size cannot become closer and closer. Hence, to obtain the weak convergence of $\left\{S_{n}(t), t \in[0,1]\right\}$ in $(D([0,1]), d)$, it is necessary to impose additional conditions on $\left(X_{i, n}\right)$ (see Rem. 6 in [8] for more details). Since the sample paths of the Wiener process are continuous, it is natural to make these assumptions on the array $\left(X_{i, n}^{(\eta)}\right)$ only.

Theorem 3. Let $\left(X_{i, n}\right)_{i \in \mathbb{N}, n \in \mathbb{N}}$ be a stationary array of associated square integrable and centered real-valued random variables. Suppose that $\mathbb{E}\left(X_{0, n}^{2}\right)$ tends to zero as $n$ tends to infinity. Assume moreover that Conditions (2.2) and (2.3) hold and that

$$
\lim _{\eta \rightarrow 0} \limsup _{n \rightarrow+\infty} n \sum_{r=1}^{n} \operatorname{Cov}\left(X_{0, n}^{(\eta)}, X_{r, n}^{(\eta)}\right)=0 .
$$

Suppose that there exists a distribution function $G$, continuous at 0 , such that for any point of continuity $x$ of $G$

$$
\lim _{\eta \rightarrow 0} \limsup _{n \rightarrow+\infty} n \mathbb{E}\left(X_{0, n}^{(\eta) 2} \mathbb{I}_{X_{0, n}^{(\eta)} \leq x}\right)=G(x) .
$$

Then the sequence $\left\{S_{n}(t), t \in[0,1]\right\}$ converges in distribution to the Lévy process of law $\mu_{F}$ in the space $(D([0,1]), d)$. The function $F$ is given by $F(x)=G(x)+\mathbb{1}_{x \geq 0}(F(\infty)-G(\infty))$.

Remark. Conditions (2.2), (2.3) and (2.8) imply the tightness of the sequence of process $\left\{S_{n}(t), t \in[0,1]\right\}$ in the space $(D([0,1]), d)$.

Remark. If $N_{a, 0}(X)=1$, then $N_{0}^{(a)}(X)=1$ (cf. Sect. 4, the inequality (4.6) for a justification). In this case, the sum $S_{n}(1)$ admits an asymptotic independent representation by blocs of length 1 . In other words, if $N_{a, 0}(X)=1$ then the set $\mathcal{E}$ defined in (1.3) is nonempty and equals to $\mathbb{N}^{*}$ (we refer the reader to Cor. 2 below and to its proof for a justification of this remark), hence one may expect a limit theorem under conditions close to that obtained for independent sequences. Those ideas were first mentioned by [23] in order to prove the convergence in distribution of $S_{n}(1)$. 
Corollary 2. Let $\left(X_{i, n}\right)_{i \in \mathbb{N}, n \in \mathbb{N}}$ be as an in Theorem 3. Suppose that

$$
\lim _{n \rightarrow+\infty} n \sum_{r=1}^{n} \operatorname{Cov}\left(X_{0, n}, X_{r, n}\right)=0 .
$$

Let $F$ be the distribution function of some finite measure. Then the following statements are equivalent (i) For any continuity point $x$ of $F$ (including $+\infty$ )

$$
\lim _{n \rightarrow+\infty} n \mathbb{E}\left(X_{0, n}^{2} \mathbb{I}_{X_{0, n} \leq x}\right)=F(x) .
$$

(ii) The sequence of process $\left\{S_{n}(t), t \in[0,1]\right\}$ converges in distribution in $D([0,1])$ to the Lévy process of law $\mu_{F}$.

Theorem 3 contains both the Gaussian and the Poisson limits as shown in the following proposition (we omit the proof, which follows immediately from Th. 3).

Proposition 3. Let $\left(X_{i, n}\right)_{i \in \mathbb{N}, n \in \mathbb{N}}$ be as an in Theorem 3. Assume that Conditions (2.2), (2.3) and (2.8) are satisfied. Suppose moreover that there exists a positive real number $\lambda$ such that

$$
\lim _{\eta \rightarrow 0} \limsup _{n \rightarrow+\infty} n \mathbb{E}\left(X_{0, n}^{(\eta) 2} \mathbb{I}_{X_{0, n}^{(\eta)} \leq x}\right)=\lambda \mathbb{I}_{1 \leq x}
$$

for any $x \neq 1$, including $+\infty$. Then the sequence of processes $\left\{S_{n}(t), t \in[0,1]\right\}$ converges in distribution in the space $(D([0,1]), d)$ to the Lévy process of law $\mu_{F}$. The characteristic function $\hat{\mu}$ of $\mu_{F}$ is given by

$$
\hat{\mu}(z)=\exp \left(-\frac{1}{2} \sigma^{2} z^{2}+\lambda\left(\mathrm{e}^{i z}-1-i z\right)\right),
$$

where $\sigma^{2}=F(\infty)-\lambda$ and $F(\infty)$ is the positive constant defined in (2.3).

Remark. Clearly $\sigma^{2}$ fulfills

$$
\sigma^{2}=\lim _{\eta \rightarrow 0} \limsup _{n \rightarrow+\infty}\left(\operatorname{Var} S_{n}(1)-n \mathbb{E}\left(X_{0, n}^{(\eta) 2}\right)\right)
$$

2.2.1. Convergence to Wiener processes

Corollary 3. Let $\left(X_{i, n}\right)_{i \in \mathbb{N}, n \in \mathbb{N}}$ be a stationary array of associated square-integrable and centered random variables. Suppose that $\mathbb{E}\left(X_{0, n}^{2}\right)$ tends to zero as $n$ tends to infinity and that Conditions (2.2) and (2.3) are satisfied with $F(\infty)=1$. If moreover

$$
\lim _{n \rightarrow+\infty} n \mathbb{E}\left(X_{0, n}^{2} \mathbb{I}_{\left|X_{0, n}\right| \geq \epsilon}\right)=0 \quad \text { for all } \quad \epsilon>0
$$

then $\left\{S_{n}(t), t \in[0,1]\right\}$ converges in distribution in $(D([0,1]), d)$ to the standard Wiener process.

For stationary and centered associated sequence with $\sum_{r=1}^{\infty} \operatorname{Cov}\left(X_{0}, X_{r}\right)<+\infty$, Corollary 3 applied to the arrays $X_{i, n}=X_{i} / \sqrt{\operatorname{Var} S_{n}}$ leads to the CLT theorem already proved in [24].

2.2.2. Convergence to Poisson processes

Corollary 4. Let $\left(X_{i, n}\right)_{i \in \mathbb{N}, n \in \mathbb{N}}$ be a stationary array of associated square-integrable and centered random variables. Assume that $\mathbb{E}\left(X_{0, n}^{2}\right)$ tends to zero as $n$ tends to infinity and consider the limits

$$
\lim _{n \rightarrow+\infty} n \mathbb{E}\left(X_{0, n}^{2}\left(1 \wedge\left|X_{0, n}-1\right|\right)\right)=0 \quad \text { and } \quad \lim _{n \rightarrow+\infty} n \mathbb{E}\left(X_{0, n}^{2}\right)=\lambda
$$


1. If (2.2), (2.8) and (2.14) hold, then (2.9) holds.

2. Suppose moreover that Condition (2.9) holds. Then, the process $\left\{S_{n}(t), t \in[0,1]\right\}$ converges in distribution in $(D([0,1]), d)$ to the centered Poisson process $\{\mathcal{P}(\lambda t)-\lambda t, t \in[0,1]\}$ (i.e. the Lévy process with distribution function $F=\lambda \mathbb{1}_{[1,+\infty[}$ ) if and only if (2.14) holds.

The convergence in distribution of $S_{n}(1)$ to a Poisson limit was established in [23], under the Conditions (2.9) and (2.14).

\subsection{On the convergence of Bernoulli random variables}

Let $\left(Y_{i, n}\right)$ be an array of Bernoulli distributed random variables with parameter $p_{n}$ such that $n p_{n}$ is bounded and define the centered triangular array $X_{i, n}$ by $X_{i, n}=Y_{i, n}-p_{n}$. We are interested in the asymptotic behavior of the process $S_{n}(Y, t)=S_{n}(t)+[n t] p_{n}=Y_{1, n}+\cdots+Y_{[n t], n}$, in the context of association.

For $\eta$ sufficiently small, we can choose $n$ large enough such that

$$
f^{(\eta)}\left(-p_{n}\right)=0, \quad f^{(\eta)}\left(1-p_{n}\right)=1-p_{n}-\eta, \quad f^{(\eta)}\left(X_{0, n}\right)=\left(1-p_{n}-\eta\right) Y_{0, n}
$$

We first deduce from those estimations that $N_{a, 0}(X)=N_{0}^{(a)}(X)=N_{0}(Y)$. Let us now describe the conditions on the sequence $\left(Y_{i, n}\right)$ under which the requirements of Theorem 2 are satisfied. From (2.15), we infer that (2.2), $(2.3),(2.5)$ and $(2.6)$ are satisfied as soon as

$$
\begin{gathered}
\lim _{N \rightarrow N_{0}(Y)} \limsup _{n \rightarrow+\infty}\left|n \operatorname{Var}\left(Y_{0, n}\right)+2 n \sum_{r=1}^{N-1} \operatorname{Cov}\left(Y_{0, n}, Y_{r, n}\right)-F(\infty)\right|=0 \\
\text { and } \lim _{N \rightarrow N_{0}(Y)} \limsup _{n \rightarrow+\infty} n \sum_{r=N}^{n} \operatorname{Cov}\left(Y_{0, n}, Y_{r, n}\right)=0 .
\end{gathered}
$$

Suppose moreover that, there exists a BV function $F_{N}$ such that for any $x$, point of continuity of $F_{N}$,

$$
\lim _{n \rightarrow \infty} n \mathbb{E}\left(S_{N, n}^{2}(Y, 1) \mathbb{I}_{S_{N, n}(Y, 1) \leq x}\right)-n \mathbb{E}\left(S_{N-1, n}^{2}(Y, 1) \mathbb{I}_{S_{N-1, n}(Y, 1) \leq x}\right)=F_{N}(x)
$$

then some elementary estimations based on (2.15) show that Condition $B^{\prime}(N)$ holds. Moreover 0 is a continuity point of $F_{N}$ since $S_{N, n}(Y, 1)$ is integer-valued. Combining these remarks with Theorem 2, we obtain the following corollary.

Corollary 5. Let $\left(Y_{i, n}\right)$ be an array of Bernoulli-distributed random variables with parameter $p_{n}$ such that $n p_{n}$ is bounded. Assume that (2.16) and (2.17) are satisfied, and that there exists a nondecreasing sequence of positive integers $\left(N_{i}\right)_{i \in \mathbb{N}^{*}}$ converging to $N_{0}(Y)$ such that (2.18) holds for any $N_{i}$. Then the finite dimensional distributions of the process $\left\{S_{n}(t), t \in[0,1]\right\}$ converges in distribution to those of the Lévy process of the purely non-Gaussian law $\mu_{G}$, where $G$ is the weak limit of $\left(F_{N_{i}}\right)$ in the sense of (2.7) and $G(\infty)=F(\infty)$.

Remark. Assume that $n p_{n}$ converges to some positive constant $\lambda$. Then under the assumptions of Corollary 5 , $S_{n}(Y, t)$ converges to $X_{t}+\lambda$ where $X_{t}$ has law $\mu_{G}^{t}$. From (2.18), it is clear that $G$ is piecewise constant with jumps at integer points, so that the limiting distribution of $S_{n}(Y, t)$ is necessarily integer-valued coumpound Poisson.

Remark. For $m$-dependent arrays, necessary and sufficient conditions for the convergence of $S_{n}(Y, 1)$ are given in [15]. Using our notations, these conditions are equivalent to: there exists a distribution function $F_{m+1}$ such that

$$
\lim _{n \rightarrow \infty} n \mathbb{E}\left(S_{m+1, n}^{2}(Y, 1) \mathbb{I}_{S_{m+1, n}(Y, 1) \leq x}\right)-n \mathbb{E}\left(S_{m, n}^{2}(Y, 1) \mathbb{I}_{S_{m, n}(Y, 1) \leq x}\right)=F_{m+1}(x)
$$

If $N_{0}(Y)=m+1$, we obtain the same condition as in the $m$-dependent case. In that case the distribution of the variable $S_{n}(Y, 1)$ is the law of the sum $V_{1}+\cdots+V_{N}$ where $N$ is Poisson distributed with parameter 
$\lambda=\sum_{i=1}^{m+1}\left(F_{m+1}(i)-F_{m+1}(i-1)\right) / i^{2}$ and is independent of the sequence $\left(V_{k}\right)_{k \geq 1}$ which is i.i.d. with marginal distribution $\mathbb{P}\left(V_{1}=i\right)=\left(F_{m+1}(i)-F_{m+1}(i-1)\right) /\left(\lambda i^{2}\right)$. See Theorem 1.1 in [20] for more details.

Remark. Under the conditions of Corollary 5 , we can establish the joint convergence of $\left(S_{n}\left(t_{1}\right), \ldots, S_{n}\left(t_{k}\right)\right)$ for any $k$-tuple $\left(t_{1}, \ldots, t_{k}\right)$ (cf. Sect. 4.2 and Lem. 15). Therefore, there is also convergence for the point process $S_{n}(Y, A)=\sum_{i \in n A} Y_{i, n}$ indexed by Borel subsets of $[0,1]$ (see Th. 4.2 in [19]).

We now discuss the number of exceedances under association (note that the method of the proof can be extended to other dependent variables). We refer to [14] for the importance of the exceedance process in extreme value theory. Let $\left(X_{i}\right)_{1 \leq i \leq n}$ be a collection of random variables and let $S_{n}$ counts for how many $i$ 's $X_{i}$ exceeds $z_{i}$, where the levels $\left(z_{i}\right)_{1 \leq i \leq n}$ are any real numbers. A poisson approximation for the law of $S_{n}$ is appropriate whenever the probabilities of exceedance are small, as shown in the following proposition.

Proposition 4. Let $\left(X_{i}\right)_{i \in \mathbb{N}}$ be a stationary sequence of associated real-valued random variables, and $B_{1}^{3}(\mathbb{R})$ be the set of three-times continuously differentiable real-valued functions for which $\left\|h^{\prime \prime}\right\|_{\infty} \leq 1$ and $\left\|h^{(3)}\right\|_{\infty} \leq 1$. Let $\left(z_{i}\right)_{i \in \mathbb{N}}$ be real numbers and define $p_{n}=\mathbb{P}\left(X_{1}>z_{n}\right)$ and $\lambda_{n}=n p_{n}$. Then

$$
\sup _{h \in B_{1}^{3}(\mathbb{R})}\left|\mathbb{E} h\left(\sum_{i=1}^{n}\left(\mathbb{I}_{X_{i}>z_{n}}-p_{n}\right)\right)-\mu_{1, \lambda_{n}}(h)\right| \leq 9 n p_{n}^{2}+2 n \sum_{r=1}^{n-1} \operatorname{Cov}\left(\mathbb{I}_{X_{0}>z_{n}}, \mathbb{I}_{X_{r}>z_{n}}\right),
$$

where $\mu_{1, \lambda_{n}}$ denotes the centered Poisson law $\mathcal{P}\left(\lambda_{n}\right)-\lambda_{n}$.

Proposition 4 is based on Lindeberg's method. Poisson approximation for dependent trials was discussed by Chen since 1975 ( $c f .[6]$ ). Chen's method is the adaptation to the Poisson distribution of Stein's differential method for the normal distribution ( $c f$. [29]). We refer to the monograph [2] Barbour for the Stein-Chen method as well as for the wide applicability of the Poisson approximations.

Let $\left(X_{i}\right)_{i \in \mathbb{N}}$ be a standardized stationary associated normal sequence, which means that the function $r(k)=$ $\operatorname{Cov}\left(X_{0}, X_{k}\right)$ is always positive (see [26]). Let $\left(z_{n}\right)$ and $\lambda_{n}$ be real numbers such that $\lambda_{n}=n\left(1-\Phi\left(z_{n}\right)\right.$ ), where $\Phi$ is the distribution function of the standard normal law. Suppose that $\sup _{n} \lambda_{n}<\infty$. An estimation of $\left|\operatorname{Cov}\left(\mathbb{I}_{X_{i}>z_{n}}, \mathbb{I}_{X_{j}>z_{n}}\right)\right|$ as it is done in [13] together with Proposition 4 leads to the following: If $r(k) \geq 0$ for each $k$ and $r(k) \leq C / \ln k$ for $k \geq 2$, then

$$
\sup _{h \in B_{1}^{3}(\mathbb{R})}\left|\mathbb{E} h\left(\sum_{i=1}^{n}\left(\mathbb{I}_{X_{i}>z_{n}}-p_{n}\right)\right)-\mu_{1, \lambda_{n}}(h)\right|=O\left(n^{-(1+\rho) /(1+\rho)}(\ln n)^{-\rho /(1+\rho)}+\frac{\ln n}{n} \sum_{k=1}^{n}|r(k)|\right),
$$

where $\rho=\max (r(1), r(2), \ldots)$. Similar results are contained in [13], but their approach is different from ours (see also [21]).

\section{Proofs For WEAKLY DEPENDENT SEQUENCES}

\subsection{Proofs of Theorem 1 and Corollary 1}

We first recall a technical result which is the main tool for the proof of Theorem 1.

Lemma 1. Let $\left(Y_{i, n}\right)_{i \in \mathbb{N}, n \in \mathbb{N}}$ and $\left(\epsilon_{i, n}\right)_{i \in \mathbb{N}, n \in \mathbb{N}}$ be two independent arrays of i.i.d and centered real valued random variables, such that $\lim _{n \rightarrow+\infty} \mathbb{E}\left(Y_{0, n}^{2}\right)=\lim _{n \rightarrow+\infty} \mathbb{E}\left(\epsilon_{0, n}^{2}\right)=0$. Let $K_{n}$ be a positive integer. Let $x_{0} \leq \ldots \leq x_{N}$ be a finite grid and define $f_{j}(t)=t^{2} \mathbb{I}_{x_{j}<t \leq x_{j+1}}$. Then, for any function $h$ three times differentiable 
with $\left\|h^{\prime \prime}\right\|_{\infty}<\infty,\left\|h^{(3)}\right\|_{\infty}<\infty$, we have

$$
\begin{aligned}
\left|\mathbb{E}\left(h\left(\sum_{i=1}^{K_{n}} Y_{i, n}\right)-h\left(\sum_{i=1}^{K_{n}} \epsilon_{i, n}\right)\right)\right| \leq & \left\|h^{\prime \prime}\right\|_{\infty} K_{n} \sum_{j=0}^{N-1}\left|\mathbb{E}\left(f_{j}\left(Y_{1, n}\right)\right)-\mathbb{E}\left(f_{j}\left(\epsilon_{1, n}\right)\right)\right| \\
& +\left\|h^{\prime \prime}\right\|_{\infty} K_{n} \mathbb{E}\left(Y_{1, n}^{2} \mathbb{I}_{\left.\left.Y_{1, n} \notin\right] x_{0}, x_{N}\right]}\right)+\left\|h^{\prime \prime}\right\|_{\infty} K_{n} \mathbb{E}\left(\epsilon_{1, n}^{2} \mathbb{I}_{\left.\left.\epsilon_{1, n} \notin\right] x_{0}, x_{N}\right]}\right) \\
& +K_{n} \sum_{j=0}^{N-1} \mathbb{E}\left(f_{j}\left(Y_{1, n}\right)\left[\left\|h^{\prime \prime}\right\|_{\infty} \wedge\left\|h^{(3)}\right\|_{\infty}\left|Y_{1, n}-x_{j}\right|\right]\right) \\
& +K_{n} \sum_{j=0}^{N-1} \mathbb{E}\left(f_{j}\left(\epsilon_{1, n}\right)\left[\left\|h^{\prime \prime}\right\|_{\infty} \wedge\left\|h^{(3)}\right\|_{\infty}\left|\epsilon_{1, n}-x_{j}\right|\right]\right)
\end{aligned}
$$

Proof of Lemma 1. Let $x_{0} \leq \ldots \leq x_{N}$ be $N+1$ fixed real numbers. Let $g$ be a bounded function with bounded first derivative. Clearly

$$
\begin{aligned}
\mathbb{E}\left(Y_{i, n}^{2} g\left(Y_{i, n}\right)\right)= & \sum_{j=0}^{N-1} \mathbb{E}\left(Y_{i, n}^{2}\left(g\left(Y_{i, n}\right)-g\left(x_{j}\right)\right) \mathbb{1}_{x_{j}<Y_{i, n} \leq x_{j+1}}\right)+\sum_{j=0}^{N-1} \mathbb{E}\left(Y_{i, n}^{2} g\left(x_{j}\right) \mathbb{I}_{x_{j}<Y_{i, n} \leq x_{j+1}}\right) \\
& +\mathbb{E}\left(Y_{i, n}^{2} g\left(Y_{i, n}\right) \mathbb{I}_{\left.\left.Y_{i, n} \notin\right] x_{0}, x_{N}\right]}\right) .
\end{aligned}
$$

According to the properties of the function $g$, we deduce from the last equality together with the analogous one for $\mathbb{E}\left(\epsilon_{i, n}^{2} g\left(\epsilon_{i, n}\right)\right)$ that

$$
\begin{aligned}
\left|\mathbb{E}\left(Y_{i, n}^{2} g\left(Y_{i, n}\right)\right)-\mathbb{E}\left(\epsilon_{i, n}^{2} g\left(\epsilon_{i, n}\right)\right)\right| \leq & \|g\|_{\infty} \sum_{j=0}^{N-1}\left|\mathbb{E}\left(f_{j}\left(Y_{i, n}\right)\right)-\mathbb{E}\left(f_{j}\left(\epsilon_{i, n}\right)\right)\right| \\
& +\|g\|_{\infty} \mathbb{E}\left(Y_{i, n}^{2} \mathbb{I}_{\left.\left.Y_{i, n} \notin\right] x_{0}, x_{N}\right]}\right)+\|g\|_{\infty} \mathbb{E}\left(\epsilon_{i, n}^{2} \mathbb{I}_{\left.\left.\epsilon_{i, n} \notin\right] x_{0}, x_{N}\right]}\right) \\
& +\sum_{j=0}^{N-1} \mathbb{E}\left(f_{j}\left(Y_{i, n}\right)\left[2\|g\|_{\infty} \wedge\left\|g^{\prime}\right\|_{\infty}\left|Y_{i, n}-x_{j}\right|\right]\right) \\
& +\sum_{j=0}^{N-1} \mathbb{E}\left(f_{j}\left(\epsilon_{i, n}\right)\left[2\|g\|_{\infty} \wedge\left\|g^{\prime}\right\|_{\infty}\left|\epsilon_{i, n}-x_{j}\right|\right]\right) .
\end{aligned}
$$

Define the random functions $h_{i-1, i+1}^{\prime \prime}(x)=h^{\prime \prime}\left(Y_{1, n}+\ldots+Y_{i-1, n}+x+\epsilon_{i+1, n}+\ldots+\epsilon_{K_{n}, n}\right)$ and $g_{i}(x)=$ $\int_{0}^{1}(1-t) h_{i-1, i+1}^{\prime \prime}(t x) \mathrm{d} t$. From Lindeberg's decomposition and the independence of $\left(Y_{i, n}\right)$ and $\left(\epsilon_{i, n}\right)$, we obtain that

$$
\mathbb{E}\left(h\left(\sum_{i=1}^{K_{n}} Y_{i, n}\right)\right)-\mathbb{E}\left(h\left(\sum_{i=1}^{K_{n}} \epsilon_{i, n}\right)\right)=\sum_{i=1}^{K_{n}} \mathbb{E}\left(Y_{i, n}^{2} g_{i}\left(Y_{i, n}\right)\right)-\sum_{i=1}^{K_{n}} \mathbb{E}\left(\epsilon_{i, n}^{2} g_{i}\left(\epsilon_{i, n}\right)\right) .
$$

Clearly $g_{i}$ is bounded by $1 / 2\left\|h^{\prime \prime}\right\|_{\infty}$ and is $1 / 6\left\|h^{(3)}\right\|_{\infty}$-lipschitz. Lemma 1 follows from (3.2) and (3.3). 
Proof of Theorem 1. We now prove Theorem 1 and the remark below. We suppose without loss of generality that $t=1$. The direct parts of Theorem 1 and Corollary 1 follow by adapting the arguments of the proof of Theorem 1 in [8]. So we only prove the second implication. Let $\left(\epsilon_{i, n}\right)_{i \in \mathbb{N}}$ be an i.i.d. array of independent and centered random variables with marginal distribution $\mu_{F}^{1 / n}$. For two positive integers $p$ and $q$ such that $p q \leq n$, define

$$
\begin{gathered}
U_{i, n}(q)=X_{i q-q+1, n}+\ldots+X_{i q, n}, \quad V_{i, n}(q)=U_{1, n}(q)+\ldots+U_{i, n}(q) \\
\Delta_{i, n}(q)=\epsilon_{i q-q+1, n}+\ldots+\epsilon_{i q, n}, \quad \Gamma_{i, n}(p)=\Delta_{i, n}(q)+\ldots+\Delta_{p, n}(q) \\
S_{q, n}=X_{1, n}+\ldots+X_{q, n}, \quad s_{n}=\epsilon_{1, n}+\ldots+\epsilon_{n, n} .
\end{gathered}
$$

A collection of random variables $\mathcal{V}=\left(V_{1}, \ldots, V_{n}\right)$ is a coupled version of $\mathcal{U}=\left(U_{1}, \ldots, U_{n}\right)$ if the members of $\mathcal{V}$ are independent and if the random variables $U_{i}$ and $V_{i}$ have the same distribution for each $1 \leq i \leq n$. We consider $\left(\tilde{U}_{1, n}(q), \ldots, \tilde{U}_{p, n}(q)\right)$ a coupled version of $\left(U_{1, n}(q), \ldots, U_{p, n}(q)\right)$ and we define

$$
\tilde{V}_{p, n}(q)=\tilde{U}_{1, n}(q)+\ldots+\tilde{U}_{p, n}(q)
$$

In order to prove Theorem 1 , we take $q=[n / p]$ where $p$ is a fixed integer. For the proof of the remark following Theorem $1, q$ will be equal to a fixed integer $m$ and $p=[n / m]$. Clearly

$$
\left|\mathbb{E} \exp \left(i t S_{n, n}\right)-\mathbb{E} \exp \left(i t s_{n}\right)\right| \leq T_{1, n}(q)+T_{2, n}(q)+T_{3, n}(q)
$$

where

$$
\begin{aligned}
& T_{1, n}(q)=\left|\mathbb{E} \exp \left(i t S_{n, n}\right)-\mathbb{E} \exp \left(i t \tilde{V}_{p, n}(q)\right)\right| \\
& T_{2, n}(q)=\mid \mathbb{E} \exp \left(i t \tilde{V}_{p, n}(q)\right)-\mathbb{E} \exp \left(i t\left(\Gamma_{1, n}(p)\right) \mid\right. \\
& T_{3, n}(q)=\left|\mathbb{E} \exp \left(i t\left(\Gamma_{1, n}(p)\right)\right)-\mathbb{E} \exp \left(i t s_{n}\right)\right|
\end{aligned}
$$

Control of $T_{1, n}(q)$

Clearly $\mathbb{E} \exp \left(i t \tilde{V}_{p, n}(q)\right)=\left(\mathbb{E} \exp \left(i t S_{q, n}\right)\right)^{p}$ and $T_{1, n}(q)=\left|\mathbb{E} \exp \left(i t S_{n, n}\right)-\left(\mathbb{E} \exp \left(i t S_{q, n}\right)\right)^{p}\right|$. Condition $(1.1)$ (resp. (1.4)) leads to (3.5) (resp. (3.6)) below

$$
\begin{aligned}
& \lim _{p \rightarrow+\infty} \limsup _{n \rightarrow+\infty} T_{1, n}([n / p])=0 \\
& \lim _{m \rightarrow m_{0}(X)} \limsup _{n \rightarrow+\infty} T_{1, n}(m)=0 .
\end{aligned}
$$

\section{Control of $T_{2, n}(q)$}

In order to control the quantities $T_{2, n}(q)$, we use Lemma 1 with a suitable choice of the grid $x_{0} \leq \ldots \leq x_{N}$. Let $\epsilon>0$ be fixed and choose an integer $N=N_{\epsilon}$ and a subdivision $x_{0} \leq x_{1} \leq \ldots \leq x_{N}$ of continuity points of $F$ such that

$$
\left|x_{j}-x_{j+1}\right| \leq \epsilon, F\left(x_{0}\right) \leq \epsilon, \quad F(\infty)-F\left(x_{N}\right) \leq \epsilon
$$


We apply Lemma 1 with $K_{n}=p$ and $h(x)=\exp (i t x)$ (more precisely $h(x)$ is either the real or the imaginary part of $\exp (i t x))$.

$$
\begin{aligned}
\left|\mathbb{E}\left(h\left(\sum_{i=1}^{p} \tilde{U}_{i, n}(q)\right)-h\left(\sum_{i=1}^{p} \Delta_{i, n}(q)\right)\right)\right| \leq & p t^{2} \sum_{j=0}^{N-1}\left|\mathbb{E}\left(f_{j}\left(U_{1, n}(q)\right)\right)-\mathbb{E}\left(f_{j}\left(\Delta_{1, n}(q)\right)\right)\right| \\
& +p t^{2} \mathbb{E}\left(U_{1, n}^{2}(q) \mathbb{I}_{\left.\left.U_{1, n}(q) \notin\right] x_{0}, x_{N}\right]}\right) \\
& +p t^{2} \mathbb{E}\left(\Delta_{1, n}^{2}(q) \mathbb{I}_{\left.\left.\Delta_{1, n}(q) \notin\right] x_{0}, x_{N}\right]}\right) \\
& +p \sum_{j=0}^{N-1} \mathbb{E}\left(f_{j}\left(U_{1, n}(q)\right)\left[t^{2} \wedge|t|^{3}\left|U_{1, n}(q)-x_{j}\right|\right]\right) \\
& +p \sum_{j=0}^{N-1} \mathbb{E}\left(f_{j}\left(\Delta_{1, n}(q)\right)\left[t^{2} \wedge|t|^{3}\left|\Delta_{1, n}(q)-x_{j}\right|\right]\right) .
\end{aligned}
$$

The last bound together with the choice of the grid $x_{0}, x_{1}, \ldots, x_{N}$ leads to

$$
\begin{aligned}
\left|\mathbb{E}\left(h\left(\sum_{i=1}^{p} \tilde{U}_{i, n}(q)\right)-h\left(\sum_{i=1}^{p} \Delta_{i, n}(q)\right)\right)\right| \leq & p t^{2} \sum_{j=0}^{N-1}\left|\mathbb{E}\left(f_{j}\left(U_{1, n}(q)\right)\right)-\mathbb{E}\left(f_{j}\left(\Delta_{1, n}(q)\right)\right)\right| \\
& +p t^{2} \mathbb{E}\left(U_{1, n}^{2}(q) \mathbb{I}_{\left.\left.U_{1, n}(q) \notin\right] x_{0}, x_{N}\right]}\right) \\
& +p t^{2} \mathbb{E}\left(\Delta_{1, n}^{2}(q) \mathbb{I}_{\left.\left.\Delta_{1, n}(q) \notin\right] x_{0}, x_{N}\right]}\right) \\
& +|t|^{3} p \in \mathbb{E}\left(U_{1, n}^{2}(q)\right)+|t|^{3} p \in \mathbb{E}\left(\Delta_{1, n}^{2}(q)\right) .
\end{aligned}
$$

We now use the following lemma, that we prove at the end of this paragraph.

Lemma 2. Under Condition (1.2) and the previous notations, we have that

$$
\begin{aligned}
& \limsup _{p \rightarrow+\infty} \limsup _{n \rightarrow+\infty} p \mathbb{E}\left(U_{1, n}^{2}([n / p])\right)<+\infty \quad \text { and } \quad \limsup _{p \rightarrow+\infty} \limsup _{n \rightarrow+\infty} p \mathbb{E}\left(\Delta_{1, n}^{2}([n / p])\right)<+\infty \\
& \limsup _{p \rightarrow+\infty} \limsup _{n \rightarrow+\infty} p \mathbb{E}\left(U_{1, n}^{2}([n / p]) \mathbb{I}_{\left.\left.U_{1, n}([n / p]) \notin\right] x_{0}, x_{N}\right]}\right) \leq 2 \epsilon \\
& \limsup _{p \rightarrow+\infty} \limsup _{n \rightarrow+\infty} p \mathbb{E}\left(\Delta_{1, n}^{2}([n / p]) \mathbb{I}_{\left.\left.\Delta_{1, n}([n / p]) \notin\right] x_{0}, x_{N}\right]}\right) \leq 2 \epsilon \\
& \lim _{p \rightarrow+\infty} \limsup _{n \rightarrow+\infty} p \mathbb{E}\left(f_{j}\left(U_{1, n}([n / p])\right)\right)-\mathbb{E}\left(f_{j}\left(\Delta_{1, n}([n / p])\right)\right) \mid=0, \quad \text { for } 0 \leq j \leq N-1 .
\end{aligned}
$$

Under Condition (1.5) and the previous notations, we have that

$$
\begin{aligned}
& \limsup _{m \rightarrow m_{0}(X)} \limsup _{n \rightarrow+\infty}[n / m] \mathbb{E}\left(U_{1, n}^{2}(m)\right)<+\infty \text { and } \limsup _{m \rightarrow m_{0}(X)} \limsup _{n \rightarrow+\infty}[n / m] \mathbb{E}\left(\Delta_{1, n}^{2}(m)\right)<+\infty \\
& \limsup _{m \rightarrow m_{0}(X)} \limsup _{n \rightarrow+\infty}[n / m] \mathbb{E}\left(U_{1, n}^{2}(m) \mathbb{I}_{\left.\left.U_{1, n}(m) \notin\right] x_{0}, x_{N}\right]}\right) \leq 2 \epsilon \\
& \limsup _{m \rightarrow m_{0}(X)} \limsup _{n \rightarrow+\infty}[n / m] \mathbb{E}\left(\Delta_{1, n}^{2}(m) \mathbb{I}_{\left.\left.\Delta_{1, n}(m) \notin\right] x_{0}, x_{N}\right]}\right) \leq 2 \epsilon \\
& \lim _{m \rightarrow m_{0}(X)} \limsup _{n \rightarrow+\infty}[n / m]\left|\mathbb{E}\left(f_{j}\left(U_{1, n}(m)\right)\right)-\mathbb{E}\left(f_{j}\left(\Delta_{1, n}(m)\right)\right)\right|=0, \quad \text { for } 0 \leq j \leq N-1 .
\end{aligned}
$$


Since $\epsilon$ is arbitrary, Lemma 2 together with the bound (3.8) leads to

$$
\begin{gathered}
\lim _{p \rightarrow+\infty} \limsup _{n \rightarrow+\infty} T_{2, n}([n / p])=0 \\
\lim _{m \rightarrow m_{0}(X)} \limsup _{n \rightarrow+\infty} T_{2, n}(m)=0,
\end{gathered}
$$

as soon as conditions (1.2) and (1.5) hold respectively.

\section{Control of $T_{3, n}(q)$}

Clearly $\left|\mathbb{E}\left(h\left(s_{n}\right)-h\left(\Gamma_{1, n}(p)\right)\right)\right| \leq|t|(n-p q) \mathbb{E}\left|\epsilon_{1, n}\right|$ (here $h(x)=\exp ($ itx $\left.)\right)$. Since $n-p[n / p] \leq p, n-m[n / m] \leq m$ and $\lim _{n \rightarrow+\infty} \mathbb{E}\left(\epsilon_{1, n}^{2}\right)=0$, we infer that

$$
\begin{array}{r}
\lim _{n \rightarrow+\infty} T_{3, n}([n / p])=0, \\
\lim _{n \rightarrow+\infty} T_{3, n}(m)=0 .
\end{array}
$$

Theorem 1 (respectively the remark below Theorem 1) follows by combining (3.5), (3.9), (3.11) (respectively in (3.6), (3.10), (3.12)) and (3.4).

Proof of Lemma 2. In order to prove Lemma 2, we need the following result (which is proved exactly as Lemma 1 in $[8])$.

Lemma 3. If $\left(t_{n}\right)$ is a sequence decreasing to 0 as $n$ goes to $+\infty$, then, for any $x$ point of continuity of $F$, we have

$$
\lim _{n \rightarrow+\infty} \frac{1}{t_{n}} \int_{-\infty}^{x} y^{2} \mathrm{~d} \mu_{F}^{t_{n}}(y)=F(x) .
$$

We now continue the proof of Lemma 2. The distribution function of $\Delta_{1, n}(q)$ is $\mu_{F}^{q / n}$. This fact together with Lemma 3 yields, for any $x$ point of continuity of $F$,

$$
\begin{aligned}
& \lim _{n \rightarrow+\infty} \frac{n}{m} \mathbb{E}\left(\Delta_{1, n}^{2}(m) \mathbb{I}_{\Delta_{1, n}(m) \leq x}\right)=F(x) \quad \text { and } \\
& \left.\lim _{p \rightarrow+\infty} \limsup _{n \rightarrow+\infty} \mid \frac{n}{[n / p]} \mathbb{E}\left(\Delta_{1, n}^{2}([n / p])\right) \mathbb{I}_{\Delta_{1, n}([n / p]) \leq x}\right)-F(x) \mid .
\end{aligned}
$$

Those facts together with conditions (1.2) and (1.5) imply Lemma 2.

\subsection{Proof of Proposition 1}

Let $\mathcal{F}_{C}$ be the class of three-times continuously differentiable functions $f$ from $\mathbb{R}$ to $\mathbb{R}$ such that $f(0)=$ $f^{\prime}(0)=0$ and $\left\|f^{\prime \prime}\right\|_{\infty} \leq C,\left\|f^{\prime \prime \prime}\right\|_{\infty} \leq C$. The following lemma reduces the behaviour of $f\left(S_{n}(t)\right)$ to that of $f\left(S_{n}^{(\eta)}(t)\right)$ and $f\left(S_{n, \eta}(t)\right)$ for any functions $f$ in $\mathcal{F}_{C}$.

Lemma 4. Let $\left(X_{i, n}\right)$ be a triangular array of stationary and centered real valued random variables. Suppose that $\mathbb{E}\left(X_{0, n}^{2}\right)<+\infty$. Let $f$ be any function of $\mathcal{F}_{C}$. Then, for any positive integer $p$ and positive real numbers $\epsilon$ and $\eta$, the term

$$
\left|p \mathbb{E} f\left(S_{n}(1 / p)\right)-p \mathbb{E} f\left(S_{n}^{(\eta)}(1 / p)\right)-\frac{f^{\prime \prime}(0)}{2} p\left(\operatorname{Var} S_{n}(1 / p)-\operatorname{Var} S_{n}^{(\eta)}(1 / p)\right)\right|
$$


is smaller than

$2 C p \mathbb{E}\left(S_{n, \eta}^{2}(1 / p) \mathbb{I}_{\left|S_{n, \eta}(1 / p)\right| \geq \epsilon}\right)+3 C \epsilon\left(V_{n, \eta}(p)+V_{n}^{(\eta)}(p)\right)+2 C\left(p \mathbb{E}\left(S_{n, \eta}^{2}(1 / p) \mathbb{I}_{\left|S_{n, \eta}(1 / p)\right| \geq \epsilon}\right)\right)^{1 / 2}\left(V_{n}^{(\eta)}(p)\right)^{1 / 2}$

recall that $V_{n, \eta}(p)=p \operatorname{Var} S_{n, \eta}(1 / p)$ and $V_{n}^{(\eta)}(p)=p \operatorname{Var} S_{n}^{(\eta)}(1 / p)$.

We prove this lemma at the end of this section and we continue the proof of Proposition 1 . Let $x$ be a fixed point of continuity of $F$. We first suppose that $x \neq 0$. Let $\nu>0$ be small enough $(\nu<|x|)$, in such a way that $x, x-\nu, x+\nu$ have the same sign. Define $f_{x, \nu^{+}}(y)=y^{2} h_{x, \nu^{+}}(y)$ and $f_{x, \nu^{-}}(y)=y^{2} h_{x, \nu^{-}}(y)$. The functions $h_{x, \nu^{+}}$and $h_{x, \nu^{-}}$are both positive, bounded by one, three times continuously differentiable, $h_{x, \nu^{+}}$ (resp. $h_{x, \nu^{-}}$) is equal to one on $\left.]-\infty, x\right]$ (resp. $\left.\left.]-\infty, x-\nu\right]\right)$ and to zero on $[x+\nu,+\infty[(\operatorname{resp}$. [x, $+\infty[)$. Clearly $f_{x, \nu^{+}}$and $f_{x, \nu^{-}}$belong to $\mathcal{F}_{C}, f_{x, \nu^{+}}^{\prime \prime}(0)=2 h_{x, \nu^{+}}(0)=2 \mathbb{1}_{0<x}$ and $f_{x, \nu^{-}}^{\prime \prime}(0)=2 \mathbb{1}_{0<x}$.

Applying Lemma 4 to the function $f_{x, \nu^{+}}$, and using $\left(\mathcal{C}_{1}\right)$ together with $\left(\mathcal{C}_{2}\right)$, we obtain

$$
\lim _{\eta \rightarrow 0} \limsup _{p \rightarrow+\infty} \limsup _{n \rightarrow+\infty}\left|p \mathbb{E} f_{x, \nu^{+}}\left(S_{n}(1 / p)\right)-p \mathbb{E} f_{x, \nu^{+}}\left(S_{n}^{(\eta)}(1 / p)\right)-\mathbb{I}_{0<x}\left(V_{n}(p)-V_{n}^{(\eta)}(p)\right)\right|=0
$$

We first prove that (1.2) follows from (1.8). The limit (1.8), the expression of $f_{x, \nu^{+}}$and the fact that $x$ is a point of continuity of $F$, ensure that

$$
\lim _{\nu \rightarrow 0} \limsup _{\eta \rightarrow 0} \limsup _{p \rightarrow+\infty} \limsup _{n \rightarrow+\infty}\left|p \mathbb{E} f_{x, \nu^{+}}\left(S_{n}^{(\eta)}(1 / p)\right)-F(x)+\mathbb{I}_{0<x}\left(V_{n}(p)-V_{n}^{(\eta)}(p)\right)\right|=0 .
$$

Collecting (3.13) and (3.14), we obtain that

$$
\lim _{\nu \rightarrow 0} \limsup _{p \rightarrow+\infty} \limsup _{n \rightarrow+\infty}\left|p \mathbb{E} f_{x, \nu^{+}}\left(S_{n}(1 / p)\right)-F(x)\right|=0 .
$$

This limit together with the analogous one for $f_{x, \nu^{-}}$proves (1.2), for any $x$ point of continuity of $F$ not equal to 0. Conversely, the limit in (1.2) gives (3.15) for any $x$ point of continuity of $F$, which together with (3.13) proves (3.14). The limit in (3.14) and its analogous for $f_{x, \nu^{-}}$instead of $f_{x, \nu^{+}}$proves (1.8) from (1.2). Hence (1.2) and (1.8) are equivalent for any $x$ point of continuity of $F$ not equal to 0 (under $\left(\mathcal{C}_{1}\right)$ and $\left(\mathcal{C}_{2}\right)$ ).

Let us prove the limit in (1.9). We suppose, from now until the end of the proof of this proposition that (1.2) (or equivalently (1.8)) holds for any $x$ point of continuity of $F$ not equal to 0 . Let $\nu>0$ be such that $\nu$ and $-\nu$ are points of continuity of $F$. From (1.8) and the fact that $\lim _{\epsilon \rightarrow 0}(F(\epsilon)-F(-\epsilon))=\sigma^{2}$, we infer that

$$
\lim _{\nu \rightarrow 0} \limsup _{\eta \rightarrow 0} \limsup _{p \rightarrow+\infty} \limsup _{n \rightarrow+\infty}\left|p \mathbb{E}\left(S_{n}^{(\eta) 2}(1 / p) \mathbb{I}_{-\nu<S_{n}^{(\eta)}(1 / p) \leq \nu}\right)-\sigma^{2}+\left(V_{n}(p)-V_{n}^{(\eta)}(p)\right)\right|=0 .
$$

The limit in (1.9) is proved from the last limit together with Condition $\left(\mathcal{C}_{3}\right)$.

We now discuss the case when 0 is a point of continuity of $F$. This fact together with (1.9) ensure

$$
\lim _{\eta \rightarrow 0} \limsup _{p \rightarrow+\infty} \limsup _{n \rightarrow+\infty}\left(V_{n}(p)-V_{n}^{(\eta)}(p)\right)=0
$$

Let $\nu>0$ be such that $\nu$ and $-\nu$ are points of continuity of $F$, and consider the functions $f_{0, \nu^{+}}, f_{0, \nu^{-}}$(those functions are in $\mathcal{F}_{C}$ and $\left.f_{0, \nu^{+}}^{\prime \prime}(0)=2, f_{0, \nu^{-}}^{\prime \prime}(0)=0\right)$. Arguing as in (3.13) and using (3.16), we obtain

$$
\lim _{\eta \rightarrow 0} \limsup _{p \rightarrow+\infty} \limsup _{n \rightarrow+\infty}\left|p \mathbb{E} g\left(S_{n}^{(\eta)}(1 / p)\right)-p \mathbb{E} g\left(S_{n}(1 / p)\right)\right|=0, \quad \text { for } g \in\left\{f_{0, \nu^{+}}, f_{0, \nu^{-}}\right\}
$$


Suppose that (1.2) is true, then the limit in (3.15) still holds with $x=0$, this fact together with (3.17) ensures

$$
\lim _{\nu \rightarrow 0} \limsup _{\eta \rightarrow 0} \limsup _{p \rightarrow+\infty} \limsup _{n \rightarrow+\infty}\left|p \mathbb{E} f_{0, \nu^{+}}\left(S_{n}^{(\eta)}(1 / p)\right)-F(0)\right|=0 .
$$

The last limit together with the analogous one for $f_{0, \nu^{-}}$instead of $f_{0, \nu^{+}}$proves the limit in (1.8) for $x=0$. By similar arguments, we prove that (1.8) still ensures (1.2), whenever $x=0$ is a point of continuity of $F$.

Proof of Lemma 4. Since $f \in \mathcal{F}_{C}$, Taylor's formula yields, for any real numbers $x$ and $y$

$$
\begin{aligned}
f(y)-f(x)-\frac{f^{\prime \prime}(0)}{2}\left(y^{2}-x^{2}\right)= & (y-x) x \int_{0}^{1}\left[f^{\prime \prime}(t x)-f^{\prime \prime}(0)\right] \mathrm{d} t \\
& +(y-x)^{2} \int_{0}^{1}(1-t)\left[f^{\prime \prime}(x+t(y-x))-f^{\prime \prime}(0)\right] \mathrm{d} t .
\end{aligned}
$$

The last decomposition together with $S_{n}(t)=S_{n}^{(\eta)}(t)+S_{n, \eta}(t)$ and $\left\|f^{\prime \prime}\right\|_{\infty} \leq C,\left\|f^{\prime \prime \prime}\right\|_{\infty} \leq C$ implies that

$$
p\left|\mathbb{E} f\left(S_{n}(1 / p)\right)-\mathbb{E}\left(f\left(S_{n}^{(\eta)}(1 / p)\right)\right)-\frac{f^{\prime \prime}(0)}{2}\left(\operatorname{Var} S_{n}(1 / p)-\operatorname{Var} S_{n}^{(\eta)}(1 / p)\right)\right|
$$

is smaller than

$$
2 C p \mathbb{E}\left(\left|S_{n, \eta}(1 / p)\right|\left|S_{n}^{(\eta)}(1 / p)\right|\left[1 \wedge\left|S_{n}^{(\eta)}(1 / p)\right|\right]\right)+2 C p \mathbb{E}\left(S_{n, \eta}^{2}(1 / p)\left[1 \wedge\left(\left|S_{n, \eta}(1 / p)\right|+\left|S_{n}^{(\eta)}(1 / p)\right|\right)\right]\right) .
$$

Let $\epsilon>0$ be fixed. Clearly

$$
\begin{aligned}
p \mathbb{E}\left(S _ { n , \eta } ^ { 2 } ( 1 / p ) \left[1 \wedge \left(\left|S_{n, \eta}(1 / p)\right|\right.\right.\right. & \left.\left.\left.+\left|S_{n}^{(\eta)}(1 / p)\right|\right)\right]\right) \\
& \leq p \mathbb{E}\left(S_{n, \eta}^{2}(1 / p) \mathbb{I}_{\left|S_{n, \eta}(1 / p)\right| \geq \epsilon}\right)+\frac{\epsilon}{2}\left(3 p \operatorname{Var} S_{n, \eta}(1 / p)+p \operatorname{Var} S_{n}^{(\eta)}(1 / p)\right) .
\end{aligned}
$$

Analogous estimations yield

$$
\begin{aligned}
p \mathbb{E}\left(\left|S_{n, \eta}(1 / p)\right|\left|S_{n}^{(\eta)}(1 / p)\right|[1\right. & \left.\left.\wedge\left|S_{n}^{(\eta)}(1 / p)\right|\right]\right) \\
& \leq\left(p \mathbb{E}\left(S_{n, \eta}^{2}(1 / p) \mathbb{I}_{\left|S_{n, \eta}(1 / p)\right| \geq \epsilon}\right)\right)^{1 / 2}\left(p \operatorname{Var} S_{n}^{(\eta)}(1 / p)\right)^{1 / 2}+\epsilon p \operatorname{Var} S_{n}^{(\eta)}(1 / p) .
\end{aligned}
$$

Lemma 4 is proved by collecting (3.19), (3.20) and (3.21).

\subsection{Proof of Proposition 2}

We only prove the direct implication, the second one being straightforward.

Proof of (1.10). Using that

$$
\left|x_{1} \ldots x_{m}-y_{1} \ldots y_{m}\right| \leq \sum_{i=1}^{m}\left|x_{i}-y_{i}\right|, \text { for } x_{i}, y_{i} \in \mathbb{C}, \quad\left|x_{i}\right|,\left|y_{i}\right| \leq 1,
$$

together with the stationarity of the sequence, we infer that

$$
\left|\mathbb{E} \exp \left(i z \tilde{S}_{n, \eta}\right)-\exp \left(-\frac{z^{2}}{2} V_{n, \eta}(p)\right)\right| \leq p\left|\mathbb{E} \exp \left(i z S_{n, \eta}(1 / p)\right)-\exp \left(-\frac{z^{2}}{2} \operatorname{Var} S_{n, \eta}(1 / p)\right)\right| \leq I+J
$$


where

$$
\begin{aligned}
& I=p\left|\mathbb{E} \exp \left(i z S_{n, \eta}(1 / p)\right)-1+\frac{z^{2}}{2} \mathbb{E} S_{n, \eta}^{2}(1 / p)\right| \\
& J=p\left|1-\frac{z^{2}}{2} \mathbb{E} S_{n, \eta}^{2}(1 / p)-\exp \left(-\frac{z^{2}}{2} \operatorname{Var} S_{n, \eta}(1 / p)\right)\right| .
\end{aligned}
$$

Control of $I$. Since $\mathbb{E} S_{n, \eta}(1 / p)=0$ and

$$
\left|\mathrm{e}^{i y}-1-i y+\frac{y^{2}}{2}\right| \leq y^{2} \wedge|y|^{3}, \text { for any } y \in \mathbb{R}
$$

we infer that

$$
\begin{aligned}
p\left|\mathbb{E} \exp \left(i z S_{n, \eta}(1 / p)\right)-1+\frac{z^{2}}{2} \mathbb{E} S_{n, \eta}^{2}(1 / p)\right| & \leq c_{z} p \mathbb{E}\left(S_{n, \eta}^{2}(1 / p) \wedge\left|S_{n, \eta}(1 / p)\right|^{3}\right) \\
& \leq c_{z} p \mathbb{E}\left(S_{n, \eta}^{2}(1 / p) \mathbb{I}_{\left|S_{n, \eta}(1 / p)\right| \geq \epsilon}\right)+c_{z} \epsilon p \operatorname{Var} S_{n, \eta}(1 / p)
\end{aligned}
$$

for any $\epsilon>0$ and $c_{z}=\max \left(z^{2},|z|^{3}\right)$. This bound together with $\left(\mathcal{C}_{1}\right)$ and $\left(\mathcal{C}_{2}\right)$ ensures that

$$
\lim _{\eta \rightarrow 0} \limsup _{p \rightarrow+\infty} \limsup _{n \rightarrow+\infty} p\left|\mathbb{E} \exp \left(i z S_{n, \eta}(1 / p)\right)-1+\frac{z^{2}}{2} \mathbb{E} S_{n, \eta}^{2}(1 / p)\right|=0 .
$$

Control of $J$. We deduce from

$$
\left|\mathrm{e}^{x}-1-x\right| \leq x^{2} \mathrm{e}^{|x|}, \quad \text { for any } x \in \mathbb{R}
$$

that

$$
p\left|1-\frac{z^{2}}{2} \mathbb{E} S_{n, \eta}^{2}(1 / p)-\exp \left(-\frac{z^{2}}{2} \operatorname{Var} S_{n, \eta}(1 / p)\right)\right| \leq p z^{4}\left(\operatorname{Var} S_{n, \eta}(1 / p)\right)^{2} \exp \left(z^{2} \operatorname{Var} S_{n, \eta}(1 / p)\right) .
$$

The last bound together with $\left(\mathcal{C}_{2}\right)$ ensures that

$$
\lim _{\eta \rightarrow 0} \limsup _{p \rightarrow+\infty} \limsup _{n \rightarrow+\infty} p\left|1-\frac{z^{2}}{2} \mathbb{E} S_{n, \eta}^{2}(1 / p)-\exp \left(-\frac{z^{2}}{2} \operatorname{Var} S_{n, \eta}(1 / p)\right)\right|=0 .
$$

The limit in (1.10) follows by collecting (3.23), (3.25) and (3.27).

Proof of (1.11). In view of (1.1) and the statement (i) of Theorem 1, it suffices to prove that

$$
\lim _{\eta \rightarrow 0} \limsup _{p \rightarrow+\infty} \limsup _{n \rightarrow+\infty}\left|\mathbb{E} \exp \left(i z \tilde{S}_{n}^{(\eta)}\right)-\exp \left(\frac{z^{2}}{2}\left(V_{n}(p)-V_{n}^{(\eta)}(p)\right)\right) \mathbb{E} \exp \left(i z \tilde{S}_{n}\right)\right|=0 .
$$

Let $\Delta V_{n, p}^{(\eta)}=\operatorname{Var} S_{n}(1 / p)-\operatorname{Var} S_{n}^{(\eta)}(1 / p)$. From (3.22) and some elementary estimations, we infer that

$$
\begin{aligned}
& \mid \mathbb{E} \exp \left(i z \tilde{S}_{n}^{(\eta)}\right)-\exp (\left.\frac{z^{2}}{2}\left(V_{n}(p)-V_{n}^{(\eta)}(p)\right)\right) \mathbb{E} \exp \left(i z \tilde{S}_{n}\right) \mid \\
& \leq p\left|\mathbb{E} \exp \left(i z S_{n}^{(\eta)}(1 / p)\right)-\exp \left(\frac{z^{2}}{2} \Delta V_{n, p}^{(\eta)}\right) \mathbb{E} \exp \left(i z S_{n}(1 / p)\right)\right| \leq I_{1}+I_{2}+I_{3}
\end{aligned}
$$


where

$$
\begin{aligned}
& I_{1}=p\left|\mathbb{E} \exp \left(i z S_{n}^{(\eta)}(1 / p)\right)-\mathbb{E} \exp \left(i z S_{n}(1 / p)\right)-\frac{z^{2}}{2} \Delta V_{n, p}^{(\eta)}\right| \\
& I_{2}=p \frac{z^{2}}{2}\left|\Delta V_{n, p}^{(\eta)}\right|\left|1-\mathbb{E} \exp \left(i z S_{n}(1 / p)\right)\right| \\
& I_{3}=p\left|\exp \left(\frac{z^{2}}{2} \Delta V_{n, p}^{(\eta)}\right)-\left(1+\frac{z^{2}}{2} \Delta V_{n, p}^{(\eta)}\right)\right| .
\end{aligned}
$$

Control of $I_{1}$. Applying Lemma 4 to the function $h(x)=\exp (i z x)-1-i z x$ (more precisely to its real and imaginary parts) and using $\left(\mathcal{C}_{1}\right)$ and $\left(\mathcal{C}_{2}\right)$, we obtain that

$$
\lim _{\eta \rightarrow 0} \limsup _{p \rightarrow+\infty} \limsup _{n \rightarrow+\infty} p\left|\mathbb{E} \exp \left(i z S_{n}^{(\eta)}(1 / p)\right)-\mathbb{E} \exp \left(i z S_{n}(1 / p)\right)-\frac{z^{2}}{2} \Delta V_{n, p}^{(\eta)}\right|=0 .
$$

Control of $I_{2}$. Proceeding as in (3.24), we obtain that

$$
p \frac{z^{2}}{2}\left|\Delta V_{n, p}^{(\eta)}\right|\left|1-\mathbb{E} \exp \left(i z S_{n}(1 / p)\right)\right| \leq \frac{|z|^{3}}{2} p\left(\operatorname{Var} S_{n}(1 / p)+\operatorname{Var} S_{n}^{(\eta)}(1 / p)\right)\left(\operatorname{Var} S_{n}(1 / p)\right)^{1 / 2} .
$$

This bound together with $\left(\mathcal{C}_{2}\right)$ implies that

$$
\lim _{p \rightarrow+\infty} \limsup _{n \rightarrow+\infty} p \frac{z^{2}}{2}\left|\Delta V_{n, p}^{(\eta)}\right|\left|1-\mathbb{E} \exp \left(i z S_{n}(1 / p)\right)\right|=0 .
$$

Control of $I_{3}$. From (3.26) and Condition $\left(\mathcal{C}_{2}\right)$, we infer that

$$
\lim _{p \rightarrow+\infty} \limsup _{n \rightarrow+\infty} p\left|\exp \left(\frac{z^{2}}{2} \Delta V_{n, p}^{(\eta)}\right)-\left(1+\frac{z^{2}}{2} \Delta V_{n, p}^{(\eta)}\right)\right|=0 .
$$

Collecting (3.29), (3.30), (3.31) and (3.32), we obtain (3.28). Now (3.28) together with Theorem 1 implies (1.11).

Proof of (1.12). Clearly

$$
\begin{aligned}
p\left|\operatorname{Cov}\left(S_{n}^{(\eta)}(1 / p), S_{n, \eta}(1 / p)\right)\right| \leq & p \mathbb{E}\left(\left|S_{n}^{(\eta)}(1 / p) S_{n, \eta}(1 / p)\right| \mathbb{I}_{\left|S_{n, \eta}(1 / p)\right|>\nu^{2}}\right) \\
& +p \mathbb{E}\left(\left|S_{n}^{(\eta)}(1 / p)\right| \mathbb{1}_{\left|S_{n}^{(\eta)}(1 / p)\right| \geq \nu}\left|S_{n, \eta}(1 / p)\right| \mathbb{1}_{\left|S_{n, \eta}(1 / p)\right| \leq \nu^{2}}\right) \\
& +p \mathbb{E}\left(\left|S_{n}^{(\eta)}(1 / p)\right| \mathbb{1}_{-\nu<S_{n}^{(\eta)}(1 / p) \leq \nu}\left|S_{n, \eta}(1 / p)\right| \mathbb{I}_{\left|S_{n, \eta}(1 / p)\right| \leq \nu^{2}}\right) .
\end{aligned}
$$

Applying Hölder's inequality, we obtain that

$$
p \mathbb{E}\left(\left|S_{n}^{(\eta)}(1 / p) S_{n, \eta}(1 / p)\right| \mathbb{I}_{\left|S_{n, \eta}(1 / p)\right|>\nu^{2}}\right) \leq\left(p \mathbb{E}\left(S_{n}^{(\eta) 2}(1 / p)\right)\right)^{1 / 2}\left(p \mathbb{E}\left(S_{n, \eta}^{2}(1 / p) \mathbb{I}_{\left|S_{n, \eta}(1 / p)\right|>\nu^{2}}\right)\right)^{1 / 2} .
$$

The last inequality together with $\left(\mathcal{C}_{1}\right)$ and $\left(\mathcal{C}_{2}\right)$ implies that

$$
\lim _{\eta \rightarrow 0} \limsup _{p \rightarrow+\infty} \limsup _{n \rightarrow+\infty} p \mathbb{E}\left(\left|S_{n}^{(\eta)}(1 / p) S_{n, \eta}(1 / p)\right| \mathbb{1}_{\left|S_{n, \eta}(1 / p)\right|>\nu^{2}}\right)=0 .
$$


Some elementary estimations lead to

$$
p \mathbb{E}\left(\left|S_{n}^{(\eta)}(1 / p)\right| \mathbb{I}_{\left|S_{n}^{(\eta)}(1 / p)\right| \geq \nu}\left|S_{n, \eta}(1 / p)\right| \mathbb{I}_{\left|S_{n, \eta}(1 / p)\right| \leq \nu^{2}}\right) \leq \nu p \mathbb{E} S_{n}^{(\eta) 2}(1 / p) .
$$

The last bound together with $\left(\mathcal{C}_{2}\right)$ yields

$$
\lim _{\nu \rightarrow 0} \limsup _{\eta \rightarrow 0} \limsup _{p \rightarrow+\infty} \limsup _{n \rightarrow+\infty} p \mathbb{E}\left(\left|S_{n}^{(\eta)}(1 / p)\right| \mathbb{I}_{\left|S_{n}^{(\eta)}(1 / p)\right| \geq \nu}\left|S_{n, \eta}(1 / p)\right| \mathbb{I}_{\left|S_{n, \eta}(1 / p)\right| \leq \nu^{2}}\right)=0
$$

We now control the last term in (3.33). Applying Hölder's inequality, we infer that

$$
p \mathbb{E}\left(\left|S_{n}^{(\eta)}(1 / p)\right| \mathbb{I}_{-\nu<S_{n}^{(\eta)}(1 / p) \leq \nu}\left|S_{n, \eta}(1 / p)\right| \mathbb{I}_{\left|S_{n, \eta}(1 / p)\right| \leq \nu^{2}}\right) \leq\left[p \mathbb{E}\left(S_{n}^{(\eta) 2}(1 / p) \mathbb{I}_{-\nu<S_{n}^{(\eta)}(1 / p) \leq \nu}\right)\right]^{1 / 2}\left[V_{n, \eta}(p)\right]^{1 / 2}
$$

This bound together with $\left(\mathcal{C}_{2}\right)$ and $\left(\mathcal{C}_{3}\right)$ implies that

$$
\lim _{\nu \rightarrow 0} \limsup _{\eta \rightarrow 0} \limsup _{p \rightarrow+\infty} \limsup _{n \rightarrow+\infty} p \mathbb{E}\left(\left|S_{n}^{(\eta)}(1 / p)\right| \mathbb{I}_{-\nu<S_{n}^{(\eta)}(1 / p) \leq \nu}\left|S_{n, \eta}(1 / p)\right| \mathbb{I}_{\left|S_{n, \eta}(1 / p)\right| \leq \nu^{2}}\right)=0
$$

Combining (3.34), (3.35), (3.37) and (3.33) we obtain (1.12).

Proof of (1.13). From Theorem 1, (1.10) and (1.11), we infer that

$$
\lim _{\eta \rightarrow 0} \limsup _{p \rightarrow+\infty} \limsup _{n \rightarrow+\infty}\left|\exp \left(\frac{z^{2}}{2} \mathcal{V}_{n, p, \eta}\right) \mathbb{E} \exp \left(i z S_{n}\right)-\mathbb{E} \exp \left(i z \tilde{S}_{n, \eta}\right) \mathbb{E} \exp \left(i z \tilde{S}_{n}^{(\eta)}\right)\right|=0
$$

where $\mathcal{V}_{n, p, \eta}=V_{n}(p)-V_{n}^{(\eta)}(p)-V_{n, \eta}(p)$. Hence (1.13) will be proved if we prove that

$$
\limsup _{\eta \rightarrow 0} \limsup _{p \rightarrow+\infty} \operatorname{lims}_{n \rightarrow+\infty}\left(V_{n}(p)-V_{n}^{(\eta)}(p)-V_{n, \eta}(p)\right)=0
$$

This follows from (1.12) and the fact that

$$
V_{n}(p)-V_{n}^{(\eta)}(p)-V_{n, \eta}(p)=2 p \operatorname{Cov}\left(S_{n}^{(\eta)}(1 / p), S_{n, \eta}(1 / p)\right)
$$

\subsection{Sufficient conditions for $\left(\mathcal{C}_{1}\right)$}

Lemma 5. Let $\left(X_{i, n}\right)_{i \in \mathbb{N}, n \in \mathbb{N}}$ be an array of stationary and centered real valued random variables, and define $S_{n, \eta}^{*}(1 / p)=\max _{1 \leq k \leq[n / p]}\left|\sum_{i=1}^{k} X_{i, n}(\eta)\right|$. For any positive integers $N, p$ and any $\epsilon>0, \eta>0$, we have

$$
\begin{aligned}
p \mathbb{E}\left(S_{n, \eta}^{2}(1 / p) \mathbb{I}_{\left|S_{n, \eta}(1 / p)\right| \geq 2 \epsilon}\right) \leq & 8 p \sum_{k=1}^{[n / p]} \operatorname{Cov}\left(\left(S_{k-N, n}(\eta)-\epsilon\right)_{+}-\left(-S_{k-N, n}(\eta)-\epsilon\right)_{+}, X_{k, n}(\eta)\right) \\
& +16 \epsilon^{-1} N p\left(\operatorname{Var}\left(\sum_{k=1}^{[n / p]} X_{k, n}^{2}(\eta)\right)\right)^{1 / 2}\left(\mathbb{E} S_{n, \eta}^{* 2}(1 / p)\right)^{1 / 2} \\
& +16 N \epsilon^{-2} n \mathbb{E} X_{0, n}^{2}(\eta) \mathbb{E} S_{n, \eta}^{* 2}(1 / p) .
\end{aligned}
$$


Proof of Lemma 5. For any real $x$ and any positive $\epsilon$, we have $x^{2} \mathbb{I}_{|x| \geq 2 \epsilon} \leq 4(|x|-\epsilon)_{+}^{2}$, where $x_{+}=\max (x, 0)$. Hence

$$
p \mathbb{E}\left(S_{n}^{2}(1 / p) \mathbb{1}_{\left|S_{n}(1 / p)\right| \geq 2 \epsilon}\right) \leq 4 p \mathbb{E}\left(\left|S_{n}(1 / p)\right|-\epsilon\right)_{+}^{2} .
$$

We first evaluate $p \mathbb{E}\left(S_{n}(1 / p)-\epsilon\right)_{+}^{2}$. Proceeding as in [27], page 52 , we have that

$$
\left(S_{n}(1 / p)-\epsilon\right)_{+}^{2} \leq 2 \sum_{k=1}^{[n / p]}\left(S_{k-1, n}-\epsilon\right)_{+} X_{k, n}+\sum_{k=1}^{[n / p]} X_{k, n}^{2} \mathbb{I}_{S_{k, n}^{*}>\epsilon}
$$

where $S_{i, n}^{*}=\max _{1 \leq j \leq i}\left|S_{j, n}\right|$. Since $\left|\left(S_{i, n}-\epsilon\right)_{+}-\left(S_{i-1, n}-\epsilon\right)_{+}\right| \leq\left|X_{i, n}\right| \mathbb{I}_{S_{i, n}^{*}>\epsilon}$ we obtain that

$$
\begin{aligned}
p \mathbb{E}\left(S_{n}(1 / p)-\epsilon\right)_{+}^{2} \leq & 2 p \sum_{k=1}^{[n / p]} \operatorname{Cov}\left(\left(S_{k-N, n}-\epsilon\right)_{+}, X_{k, n}\right) \\
& +2 p \sum_{k=1}^{[n / p]} \sum_{i=k-N+1}^{k} \mathbb{E}\left(\left|X_{k, n} X_{i, n}\right| \mathbb{I}_{S_{n}^{*}(1 / p)>\epsilon}\right)
\end{aligned}
$$

To control the second term on right hand in (3.41), note that

$$
2 p \sum_{k=1}^{[n / p]} \sum_{i=k-N+1}^{k} \mathbb{E}\left(\left|X_{k, n} X_{i, n}\right| \mathbb{I}_{S_{n}^{*}(1 / p)>\epsilon}\right) \leq 2 N p \sum_{k=1}^{[n / p]} \mathbb{E}\left(X_{k, n}^{2} \mathbb{I}_{S_{n}^{*}(1 / p)>\epsilon}\right),
$$

due to the fact that $2\left|X_{k, n}\right|\left|X_{i, n}\right| \leq X_{k, n}^{2}+X_{i, n}^{2}$. Of course the same arguments apply to $p \mathbb{E}\left(-S_{n}(1 / p)-\epsilon\right)_{+}^{2}$, and we obtain from (3.39), (3.41) and (3.42) that

$$
\begin{aligned}
p \mathbb{E}\left(S_{n}^{2}(1 / p) \mathbb{I}_{\left|S_{n}(1 / p)\right| \geq 2 \epsilon}\right) \leq & 8 p \sum_{k=1}^{[n / p]} \operatorname{Cov}\left(\left(S_{k-N, n}-\epsilon\right)_{+}-\left(-S_{k-N, n}-\epsilon\right)_{+}, X_{k, n}\right) \\
& +16 N p \sum_{k=1}^{[n / p]} \mathbb{E}\left(X_{k, n}^{2} \mathbb{I}_{S_{n}^{*}(1 / p)>\epsilon}\right) .
\end{aligned}
$$

To prove Lemma 5, we shall apply (3.43) to the array $X_{k, n}(\eta)$. Note first that

$$
p \sum_{k=1}^{[n / p]} \mathbb{E}\left(X_{k, n}^{2}(\eta) \mathbb{I}_{S_{n, \eta}^{*}(1 / p)>\epsilon}\right) \leq I+I I
$$

where

$$
I=p \sum_{k=1}^{[n / p]} \mathbb{E}\left(\left[X_{k, n}^{2}(\eta)-\mathbb{E}\left(X_{k, n}^{2}(\eta)\right)\right] \mathbb{I}_{S_{n, \eta}^{*}(1 / p)>\epsilon}\right) \text { and } I I=n \mathbb{E}\left(X_{0, n}^{2}(\eta)\right) \mathbb{P}\left(S_{n, \eta}^{*}(1 / p)>\epsilon\right)
$$


It is not hard to see that

$$
\begin{aligned}
|I| & \leq p \mathbb{E}\left|\sum_{k=1}^{[n / p]}\left[X_{k, n}^{2}(\eta)-\mathbb{E}\left(X_{k, n}^{2}(\eta)\right)\right]\right| \mathbb{I}_{S_{n, \eta}^{*}(1 / p)>\epsilon} \\
& \leq p\left(\operatorname{Var}\left(\sum_{k=1}^{[n / p]} X_{k, n}^{2}(\eta)\right)\right)^{1 / 2}\left(\mathbb{P}\left(S_{n, \eta}^{*}(1 / p)>\epsilon\right)\right)^{1 / 2} \\
& \leq \frac{p}{\epsilon}\left(\operatorname{Var}\left(\sum_{k=1}^{[n / p]} X_{k, n}^{2}(\eta)\right)\right)^{1 / 2}\left(\mathbb{E} S_{n, \eta}^{* 2}(1 / p)\right)^{1 / 2} .
\end{aligned}
$$

To control $I I$, we apply Markov's inequality:

$$
n \mathbb{E}\left(X_{0, n}^{2}(\eta)\right) \mathbb{P}\left(S_{n, \eta}^{*}(1 / p)>\epsilon\right) \leq \frac{n}{\epsilon^{2}} \mathbb{E}\left(X_{0, n}^{2}(\eta)\right) \mathbb{E} S_{n, \eta}^{* 2}(1 / p)
$$

Collecting Inequalities (3.44), (3.45) and (3.46), we obtain the bound

$$
p \sum_{k=1}^{[n / p]} \mathbb{E}\left(X_{k, n}^{2}(\eta) \mathbb{I}_{S_{n, \eta}^{*}(1 / p)>\epsilon}\right) \leq \frac{p}{\epsilon}\left(\operatorname{Var}\left(\sum_{k=1}^{[n / p]} X_{k, n}^{2}(\eta)\right)\right)^{1 / 2}\left(\mathbb{E} S_{n, \eta}^{* 2}(1 / p)\right)^{1 / 2}+\frac{n}{\epsilon^{2}} \mathbb{E} X_{0, n}^{2}(\eta) \mathbb{E} S_{n, \eta}^{* 2}(1 / p)
$$

Lemma 5 follows by applying (3.43) to the array $\left(X_{k, n}(\eta)\right)$ and by using (3.47).

\subsection{Sufficient conditions for $\left(\mathcal{C}_{3}\right)$.}

The following lemma, which is an analogue of Lemma 3.2 in [17], is a tool in order to check the limit in (1.8). In fact it controls $p \mathbb{E} h\left(S_{n}^{(\eta)}(1 / p)\right)$.

Lemma 6. Let $\left(X_{i, n}\right)$ be a triangular array of stationary real valued random variables. Let $h$ be a fixed function of the set $\mathcal{F}_{C}$. Then, for any positive integers $p, N$ and any positive number $\eta$, we have

$$
\begin{aligned}
\left|p \mathbb{E} h\left(S_{n}^{(\eta)}(1 / p)\right)-p[n / p] \mathbb{E}\left(h\left(S_{N, n}^{(\eta)}\right)-h\left(S_{N-1, n}^{(\eta)}\right)\right)\right| & \\
& \leq C N p\left(\mathbb{E}\left(S_{N-1, n}^{(\eta)}\right)^{2}+\mathbb{E}\left(S_{N, n}^{(\eta)}\right)^{2}\right)+C p \sum_{k=0}^{[n / p]-N} \mathbb{E}\left(\left|S_{k, n}^{(\eta)} X_{k+N, n}^{(\eta)}\right|\right)
\end{aligned}
$$

Remark. A maximal inequality is useful in order to control the second term on right hand in (3.48). In fact

$$
p \sum_{k=0}^{[n / p]-N} \mathbb{E}\left(\left|S_{k, n}^{(\eta)} X_{k+N, n}^{(\eta)}\right|\right) \leq p \sum_{k=0}^{[n / p]-N} \operatorname{Cov}\left(\left|S_{k, n}^{(\eta)}\right|,\left|X_{k+N, n}^{(\eta)}\right|\right)+\mathbb{E}\left(\max _{1 \leq k \leq\left[\frac{n}{p}\right]}\left|S_{k, n}^{(\eta)}\right|\right) \frac{2 n}{\eta} \mathbb{E} X_{0, n}^{2}
$$


Proof of Lemma 6. Clearly we have the two decompositions

$$
\begin{aligned}
\mathbb{E} h\left(S_{n}^{(\eta)}(1 / p)\right)= & \mathbb{E} h\left(S_{N-1, n}^{(\eta)}\right)+\sum_{k=0}^{[n / p]-N} \mathbb{E}\left(h\left(S_{k+N, n}^{(\eta)}\right)-h\left(S_{k+N-1, n}^{(\eta)}\right)\right) \text { and }(3 \\
{[n / p] \mathbb{E}\left(h\left(S_{N, n}^{(\eta)}\right)-h\left(S_{N-1, n}^{(\eta)}\right)\right)=} & (N-1) \mathbb{E}\left(h\left(S_{N, n}^{(\eta)}\right)-h\left(S_{N-1, n}^{(\eta)}\right)\right) \\
& +\sum_{k=0}^{[n / p]-N} \mathbb{E}\left(h\left(S_{k+N, n}^{(\eta)}-S_{k, n}^{(\eta)}\right)-h\left(S_{k+N-1, n}^{(\eta)}-S_{k, n}^{(\eta)}\right)\right)
\end{aligned}
$$

Applying Taylor's expansion, we obtain that

$$
\begin{aligned}
\mathbb{E} \mid\left(h\left(S_{k+N, n}^{(\eta)}\right)\right. & \left.-h\left(S_{k+N-1, n}^{(\eta)}\right)\right)-\left(h\left(S_{k+N, n}^{(\eta)}-S_{k, n}^{(\eta)}\right)-h\left(S_{k+N-1, n}^{(\eta)}-S_{k, n}^{(\eta)}\right)\right) \mid \\
& =\mathbb{E}\left|S_{k, n}^{(\eta)} \int_{0}^{1}\left[h^{\prime}\left(S_{k+N, n}^{(\eta)}-u S_{k, n}^{(\eta)}\right)-h^{\prime}\left(S_{k+N-1, n}^{(\eta)}-u S_{k, n}^{(\eta)}\right)\right] \mathrm{d} u\right| \leq C \mathbb{E}\left|S_{k, n}^{(\eta)} X_{k+N, n}^{(\eta)}\right|
\end{aligned}
$$

Lemma 6 follows by combining (3.50), (3.51) and (3.52).

\section{Proofs For Associated SEQuences}

We first recall some well known inequalities for associated sequences. We begin with Newman's inequality (cf. [22]).

Lemma 7. Let $\left(U_{n}\right)$ be a sequence of stationary and centered associated random variables with $\mathbb{E}\left(U_{1}^{2}\right)<+\infty$. Then, for any real number $z$,

$$
\left|\mathbb{E}\left(\exp \left(i z \sum_{j=1}^{n} U_{j}\right)\right)-\prod_{j=1}^{n}\left(\mathbb{E} \exp \left(i z U_{j}\right)\right)\right| \leq \frac{z^{2}}{2}\left[\mathbb{E}\left(\sum_{i=1}^{n} U_{i}\right)^{2}-n \operatorname{Var} U_{1}\right]
$$

We next recall two maximal inequalities due to Newman and Wright (cf. [24]).

Lemma 8. Let $\left(X_{n}\right)$ be a centered sequence of associated random variables with finite second moment. Let $S_{n}=X_{1}+\ldots+X_{n}, S_{n}^{*}=\max _{0 \leq k \leq n}\left|S_{k}\right|$ and $s_{n}^{2}=\operatorname{Var}\left(S_{n}\right)$. The following inequalities hold

$$
\begin{aligned}
\mathbb{E}\left(S_{n}^{* 2}\right) & \leq 2 s_{n}^{2} \\
\mathbb{P}\left(S_{n}^{*} \geq \lambda s_{n}\right) & \leq 2 \mathbb{P}\left(\left|S_{n}\right| \geq(\lambda-\sqrt{2}) s_{n}\right), \quad \text { for any } \lambda>0 .
\end{aligned}
$$

We shall also use on several occasions the following covariance inequality due to Birkel (cf. [4]). For any function $h$ from $\mathbb{R}^{k}$ to $\mathbb{R}$, denote by $\operatorname{Lip}(h)=\sup _{x \neq y}|h(x)-h(y)| /\|x-y\|_{1}$, where $\|x-y\|_{1}=\sum_{i=1}^{n}\left|x_{i}-y_{i}\right|$.

Lemma 9. Let $\left(X_{i, n}\right)_{i \in \mathbb{N}, n \in \mathbb{N}}$ be an array of associated real valued random variables. Then for all finite subsets $I$, $J$ of $\mathbb{N}$, we have that

$$
\left|\operatorname{Cov}\left(h\left(X_{i, n}, i \in I\right), k\left(X_{j, n}, j \in J\right)\right)\right| \leq \sum_{i \in I} \sum_{j \in J} \operatorname{Lip}(h) \operatorname{Lip}(k) \operatorname{Cov}\left(X_{i, n}, X_{j, n}\right) .
$$




\subsection{Main ingredients for the proofs of Theorems 2 and $\mathbf{3}$}

In order to prove Theorems 2 and 3, we need some auxiliary results.

Lemma 10. Let $\left(X_{i, n}\right)_{i \in \mathbb{N}, n \in \mathbb{N}}$ be an array of stationary centered and associated real valued random variables, with finite second moment. Then

$$
V_{n}(p) \leq \operatorname{Var} S_{n}(1), \quad 0 \leq V_{n, \eta}(p) \leq V_{n}(p), \quad 0 \leq V_{n}^{(\eta)}(p) \leq V_{n}(p)
$$

recall that $V_{n}(p), V_{n, \eta}(p), V_{n}^{(\eta)}(p)$ are defined as in Proposition 1. Suppose moreover that Conditions (2.2), (2.3) are satisfied then

$$
\lim _{n \rightarrow+\infty} \operatorname{Var} S_{n}(1)=F(\infty), \quad \lim _{p \rightarrow+\infty} \limsup _{n \rightarrow+\infty}\left|p \mathbb{E}\left(S_{n}^{2}(1 / p)\right)-F(\infty)\right|=0
$$

Proof of Lemma 10. The random variables $X_{i, n}^{(\eta)}, X_{i, n}(\eta)$ are nondecreasing and 1-Lipshitz functions of $X_{i, n}$. We infer from $\left(\mathcal{P}_{4}\right)$ in [10] that the sequences $\left(X_{i, n}^{(\eta)}\right)_{i},\left(X_{i, n}(\eta)\right)_{i}$ are still associated. This fact together with Lemma 9 yields

$$
\begin{array}{r}
0 \leq \operatorname{Cov}\left(X_{0, n}^{(\eta)}, X_{i, n}^{(\eta)}\right) \leq \operatorname{Cov}\left(X_{0, n}, X_{i, n}\right) \\
0 \leq \operatorname{Cov}\left(X_{0, n}(\eta), X_{i, n}(\eta)\right) \leq \operatorname{Cov}\left(X_{0, n}, X_{i, n}\right)
\end{array}
$$

from which we deduce the inequalities in (4.4). We now prove the first limit in (4.5). Clearly

$$
\operatorname{Var} S_{n}(1)=n \mathbb{E}\left(X_{0, n}^{2}\right)+2 \sum_{r=1}^{n-1}(n-r) \operatorname{Cov}\left(X_{0, n}, X_{r, n}\right)
$$

Hence

$$
\left|\operatorname{Var} S_{n}(1)-n \mathbb{E}\left(X_{0, n}^{2}\right)-2 n \sum_{r=1}^{N-1} \operatorname{Cov}\left(X_{0, n}, X_{r, n}\right)\right| \leq 2 n \sum_{r=N}^{n} \operatorname{Cov}\left(X_{0, n}, X_{r, n}\right)+2 \sum_{r=1}^{N-1} r \operatorname{Cov}\left(X_{0, n}, X_{r, n}\right) .
$$

The last inequality together with Conditions $(2.2),(2.3)$ and the asymptotic negligibility of $\left(X_{0, n}\right)$ prove the first limit in (4.5). The second limit follows from analogous estimations.

Lemma 11. Let $\left(X_{i, n}, i \in \mathbb{N}, n \in \mathbb{N}\right)$ be an array of stationary centered and associated real valued random variables. Suppose that $\lim _{n \rightarrow+\infty} \mathbb{E}\left(X_{0, n}^{2}\right)=0$. If Conditions (2.2), (2.3) are satisfied then Condition (1.1) is also satisfied.

Proof of Lemma 11. We prove (1.1) for $t=1$, the proof for any $t \in[0,1]$ being unchanged. Clearly

$$
\begin{aligned}
\left|\mathbb{E}\left(\exp \left(i z S_{n, n}\right)\right)-\left(\mathbb{E}\left(\exp \left(i z S_{n}(1 / p)\right)\right)\right)^{p}\right| \leq & \left|\mathbb{E}\left(\exp \left(i z S_{n, n}\right)\right)-\left(\mathbb{E}\left(\exp \left(i z S_{p[n / p], n}\right)\right)\right)\right| \\
& +\left|\mathbb{E}\left(\exp \left(i z S_{p[n / p], n}\right)\right)-\left(\mathbb{E}\left(\exp \left(i z S_{n}(1 / p)\right)\right)\right)^{p}\right|
\end{aligned}
$$

To control the first term on right hand, write

$$
\left|\mathbb{E}\left(\exp \left(i z S_{n, n}\right)\right)-\left(\mathbb{E}\left(\exp \left(i z S_{p[n / p], n}\right)\right)\right)\right| \leq|z| p \mathbb{E}\left|X_{0, n}\right|
$$


To control the second term on right hand in (4.10), we use Lemma 7

$$
\begin{aligned}
\left|\mathbb{E}\left(\exp \left(i z S_{p[n / p], n}\right)\right)-\left(\mathbb{E}\left(\exp \left(i z S_{n}(1 / p)\right)\right)\right)^{p}\right| & \leq \frac{z^{2}}{2}\left[\operatorname{Var}\left(S_{p[n / p], n}\right)-p \operatorname{Var} S_{n}(1 / p)\right] \\
& \leq \frac{z^{2}}{2}\left[\operatorname{Var}\left(S_{n, n}\right)-p \operatorname{Var} S_{n}(1 / p)\right] .
\end{aligned}
$$

the last inequality is true since $\left(\operatorname{Var}\left(S_{k, n}\right)\right)_{k}$ is nondecreasing, due to the association. Now, collecting Inequalities (4.10), (4.11), (4.12) and using Lemma 10 together with the asymptotic negligebility of $\left(X_{0, n}\right)$, we infer that (1.1) of Theorem 1 holds. Lemma 11 is then proved.

The following lemma discusses the conditions under which associated sequences satisfy $\left(\mathcal{C}_{1}\right)$.

Lemma 12. Let $\left(X_{i, n}\right)_{i \in \mathbb{N}, n \in \mathbb{N}}$ be an array of stationary centered and associated real valued random variables, with finite second moment. If Conditions (2.2), (2.3) are satisfied, then for any $\epsilon>0$,

$$
\lim _{\eta \rightarrow 0} \limsup _{p \rightarrow+\infty} \limsup _{n \rightarrow+\infty} p \mathbb{E}\left(S_{n, \eta}^{2}(1 / p) \mathbb{I}_{\left|S_{n, \eta}(1 / p)\right| \geq 2 \epsilon}\right)=0 .
$$

The limit in (4.13) remains true if instead of $\lim _{\eta \rightarrow 0} \limsup _{p \rightarrow+\infty}$, we write $\lim _{p \rightarrow+\infty} \limsup _{\eta \rightarrow 0}$.

Proof of Lemma 12. We start from (3.38). We first control the first term on right hand in (3.38). Since the nondecreasing function $x \rightarrow(x-\epsilon)_{+}$is 1-Lipshitz, the association property (cf. Lem. 9) and the stationarity lead to

$$
\begin{aligned}
p \sum_{k=1}^{[n / p]} \operatorname{Cov}\left(\left(S_{k-N, n}(\eta)-\epsilon\right)_{+}, X_{k, n}(\eta)\right) & \leq p \sum_{k=1}^{[n / p]} \operatorname{Cov}\left(S_{k-N, n}(\eta), X_{k, n}(\eta)\right) \\
& \leq n \sum_{r=N}^{[n / p]} \operatorname{Cov}\left(X_{r, n}(\eta), X_{0, n}(\eta)\right) .
\end{aligned}
$$

Since the sequence $\left(-X_{r, n}\right)$ is still associated, the same bound is true for

$$
p \sum_{k=1}^{[n / p]} \operatorname{Cov}\left(\left(-S_{k-N, n}(\eta)-\epsilon\right)_{+},-X_{k, n}(\eta)\right) .
$$

We now control the second term on right hand in (3.38). The function $x \rightarrow f_{\eta}^{2}(x)$ being $2 \eta$-lipshitz, Lemma 9 applied to the associated sequence $\left(X_{i, n}(\eta)\right)_{i}$ leads to

$$
\left|\operatorname{Cov}\left(X_{i, n}^{2}(\eta), X_{j, n}^{2}(\eta)\right)\right| \leq 4 \eta^{2} \operatorname{Cov}\left(X_{i, n}(\eta), X_{j, n}(\eta)\right), \quad \text { for } i, j \in \mathbb{N} .
$$

Hence $\operatorname{Var}\left(\sum_{k=1}^{[n / p]} X_{k, n}^{2}(\eta)\right) \leq 4 \eta^{2} \operatorname{Var}\left(S_{n, \eta}(1 / p)\right)$, which implies that

$$
\left(p \operatorname{Var}\left(\sum_{k=1}^{[n / p]}\left(X_{k, n}^{2}(\eta)\right)\right)\right)^{1 / 2} \leq 2 \eta \sqrt{p V_{n, \eta}(p)}
$$

(recall that $V_{n, \eta}(p)=p \operatorname{Var} S_{n, \eta}(1 / p)$ ). Finally, we control the last term on right hand in (3.38). From Lemma 8 and some elementary estimations, we infer that

$$
n \mathbb{E}\left(X_{0, n}^{2}(\eta)\right) \leq n \mathbb{E}\left(X_{0, n}^{2}\right), \quad \text { and } \quad \mathbb{E}\left(S_{n, \eta}^{* 2}(1 / p)\right) \leq 2 \frac{V_{n, \eta}(p)}{p} .
$$


Inequalities (4.14), (4.15), (4.16) and (3.38) imply that

$$
\begin{aligned}
& p \mathbb{E}\left(S_{n, \eta}^{2}(1 / p) \mathbb{I}_{\left|S_{n, \eta}(1 / p)\right| \geq 2 \epsilon}\right) \\
& \leq 16 n \sum_{r=N}^{[n / p]} \operatorname{Cov}\left(X_{r, n}(\eta), X_{0, n}(\eta)\right)+32 \sqrt{2} \epsilon^{-1} N \eta V_{n, \eta}(p)+16 N \epsilon^{-2} n \mathbb{E}\left(X_{0, n}^{2}\right) \frac{V_{n, \eta}(p)}{p} .
\end{aligned}
$$

This bound together with Lemma 10 implies Lemma 12.

We now discuss sufficient Conditions for $\left(\mathcal{C}_{3}\right)$ and $(1.8)$ under association.

Lemma 13. Let $\left(X_{i, n}\right)_{i \in \mathbb{N}, n \in \mathbb{N}}$ be an array of stationary centered, associated real valued random variables, with finite second moment such that $\mathbb{E} X_{0, n}^{2}$ tends to 0 as $n$ goes to infinity. Suppose that there exists a nondecreasing sequence of positive integers $\left(N_{i}\right)_{i \in \mathbb{N}^{*}}$ converging to $N_{0}^{(a)}(X)$ such that $B^{\prime}\left(N_{i}\right)$ holds for any $i$ in $\mathbb{N}^{*}$. Suppose moreover that Conditions (2.5) and (2.6) hold. Then there exists a distribution function $G$ fulfilling (2.7) and such that

$$
\lim _{\eta \rightarrow 0} \limsup _{p \rightarrow+\infty} \limsup _{n \rightarrow+\infty}\left|p \mathbb{E}\left(S_{n}^{(\eta) 2}(1 / p) \mathbb{I}_{S_{n}^{(\eta)}(1 / p) \leq x}\right)-G(x)\right|=0,
$$

for any $x$ point of continuity of $G$.

Proof of Lemma 13. We use the same arguments as in the end of the proof of Proposition 1 in [8]. Condition $\mathrm{B}^{\prime}(\mathrm{N})$ and the fact that $n \mathbb{E} S_{N, n}^{(\eta) 2}$ is uniformly bounded over $n$ and $\eta$ imply that, for any bounded and continuous function $g$,

$$
\lim _{\eta \rightarrow 0} \limsup _{n \rightarrow+\infty}\left|n \mathbb{E}\left(S_{N, n}^{(\eta) 2} g\left(S_{N, n}^{(\eta)}\right)\right)-n \mathbb{E}\left(S_{N-1, n}^{(\eta) 2} g\left(S_{N-1, n}^{(\eta)}\right)\right)-\int g(x) \mathrm{d} F_{N}(x)\right|=0
$$

On the other hand, combining (3.49), (4.3) and (4.1) we obtain that

$$
p \sum_{k=0}^{[n / p]} \mathbb{E}\left(\left|S_{k, n}^{(\eta)} X_{k+N, n}^{(\eta)}\right|\right) \leq n \sum_{k=N}^{n} \operatorname{Cov}\left(X_{0, n}^{(\eta)}, X_{k, n}^{(\eta)}\right)+\frac{2 n}{\eta} \mathbb{E} X_{0, n}^{2} \sqrt{2 \operatorname{Var}\left(S_{n}^{(\eta)}(1 / p)\right)}
$$

This bound together with (4.18), Lemmas 6 and 10 and the asymptotic negligebility of $\mathbb{E} S_{N, n}^{(\eta) 2}$, implies that

$$
\limsup _{\eta \rightarrow 0} \limsup _{p \rightarrow+\infty} \limsup _{n \rightarrow+\infty} \mid p \mathbb{E}\left(S_{n}^{(\eta) 2}(1 / p) g\left(S_{n}^{(\eta)}(1 / p)\right)-\int g(x) d F_{N}(x) \mid \leq C R^{(a)}(N)\right.
$$

where $g$ is three times differentiable with compactly supported derivatives, $\left(R^{(a)}(N)\right)_{N}$ denotes the nonincreasing sequence defined as in (2.4) and $C$ is a positive constant. Let $\left(N_{i}\right)_{i}$ be a nondecreasing sequence converging to $N_{0}^{(a)}(X)$. From (4.19), we infer that

$$
\text { for } j \geq i, \quad\left|\int g(x) \mathrm{d} F_{N_{i}}(x)-\int g(x) \mathrm{d} F_{N_{j}}(x)\right| \leq 2 C R^{(a)}\left(N_{i}\right)
$$

From this inequality and Condition (2.5), we infer that $\int g(x) d F_{N_{i}}(x)$ converges to some limit $L(g)$. This fact together with (4.19) ensures that

$$
\lim _{\eta \rightarrow 0} \limsup _{p \rightarrow+\infty} \limsup _{n \rightarrow+\infty} \mid p \mathbb{E}\left(S_{n}^{(\eta) 2}(1 / p) g\left(S_{n}^{(\eta)}(1 / p)\right)-L(g) \mid=0\right.
$$


The last limit proves that $L(g) \geq 0$ if $g$ is nonnegative, $L(f+g)=L(g)+L(f)$ and $L(\alpha f)=\alpha L(f)$. From $(2.5)$ and (2.6), we infer that $L(1)$ is finite. Using Riesz's representation theorem, we conclude that there exists a distribution function $G$, for which $L(g)=\int g \mathrm{~d} G$ for any $g$ as defined in (4.19). This fact together with (4.20) implies that

$$
\lim _{\eta \rightarrow 0} \limsup _{p \rightarrow+\infty} \limsup _{n \rightarrow+\infty}\left|p \mathbb{E}\left(S_{n}^{(\eta) 2}(1 / p) g\left(S_{n}^{(\eta)}(1 / p)\right)\right)-\int g \mathrm{~d} G\right|=0 .
$$

From estimations similar to those used in the inequality (3.1) in Section 3.1 in [8], we infer that (4.21) still holds for $g(y)=\mathbb{1}_{y \leq x}$ where $x$ is a point of continuity of $G$. Lemma 13 is proved.

\subsection{A tool for the convergence of the finite dimensional distributions under association}

We consider the following inequality, which is more general than association,

$$
\left|\operatorname{Cov}\left(h\left(X_{i, n}, i \in I\right), k\left(X_{j, n}, j \in J\right)\right)\right| \leq \sum_{i \in I} \sum_{j \in J} \operatorname{Lip}(h) \operatorname{Lip}(k)\left|\operatorname{Cov}\left(X_{i, n}, X_{j, n}\right)\right|,
$$

for all disjoint subsets $I, J$ of $\mathbb{N}$ and for all real valued functions $h, k$ such that $\operatorname{Lip}(h)$ and $\operatorname{Lip}(k)$ are finite; recall that $\operatorname{Lip}(h)$ is defined as in Lemma 9 .

Gaussian, associated or negatively dependent random variables fulfill the inequality (4.22) (see for instance $[4,5]$ or $[9])$. Recall that the triangular array $\left(X_{i, n}\right)_{i \in \mathbb{N}}$ is negatively dependent if for each $n$ and for all coordinatewise nondecreasing real-valued functions $h$ and $k$

$$
\operatorname{Cov}\left(h\left(X_{i, n}, i \in A\right), k\left(X_{i, n}, i \in B\right)\right) \leq 0,
$$

holds for all finite and disjoint subsets $A$ and $B$ of $\mathbb{N}$. We refer to [18] for more about this notion of negative dependence.

Lemma 14. Let $\left(U_{i, n}\right)_{i, n}$ be a triangular array of random variables having finite variance and satisfying (4.22) for each fixed $n$. Let $N$ be a fixed positive integer. Suppose that for each $1 \leq i \leq N,\left(U_{i, n}\right)_{n}$ converges in distribution to some random variable $Y_{i}$. If moreover

$$
\lim _{n \rightarrow+\infty} \operatorname{Cov}\left(U_{i, n}, U_{j, n}\right)=0, \text { for all } i \neq j
$$

then $\left(U_{1, n}, \ldots, U_{N, n}\right)$ converges in distribution to the $N$-tuple with independent components $\left(Y_{1}, \ldots, Y_{N}\right)$.

Proof of Lemma 14. Let $\phi_{n}\left(\alpha_{1}, \ldots, \alpha_{N}\right), \phi_{j, n}(\alpha)$ and $\tilde{\phi}_{j}(\alpha)$ be the characteristic functions of $\left(U_{1, n}, \ldots, U_{N, n}\right)$, $U_{j, n}$ and $Y_{j}$, respectively. We deduce, using the inequality (4.22)

$$
\left|\phi_{n}\left(\alpha_{1}, \ldots, \alpha_{N}\right)-\prod_{j=1}^{N} \phi_{j, n}\left(\alpha_{j}\right)\right| \leq 4 \sum_{1 \leq i \neq j \leq N}\left|\alpha_{i} \alpha_{j}\right|\left|\operatorname{Cov}\left(U_{i, n}, U_{j, n}\right)\right|
$$

(the analogous inequality under association is stated in Lem. 7). Some elementary estimations (cf. (3.22)) lead to

$$
\left|\prod_{j=1}^{N} \phi_{j, n}\left(\alpha_{j}\right)-\prod_{j=1}^{N} \tilde{\phi}_{j}\left(\alpha_{j}\right)\right| \leq \sum_{1 \leq j \leq N}\left|\phi_{j, n}\left(\alpha_{j}\right)-\tilde{\phi}_{j}\left(\alpha_{j}\right)\right| .
$$

Collecting (4.24) and (4.25), we obtain that

$$
\left|\phi_{n}\left(\alpha_{1}, \ldots, \alpha_{N}\right)-\prod_{j=1}^{N} \tilde{\phi}_{j}\left(\alpha_{j}\right)\right| \leq 4 \sum_{1 \leq i \neq j \leq N}\left|\alpha_{i}\right|\left|\alpha_{j}\right|\left|\operatorname{Cov}\left(U_{i, n}, U_{j, n}\right)\right|+\sum_{1 \leq j \leq N}\left|\phi_{j, n}\left(\alpha_{j}\right)-\tilde{\phi}_{j}\left(\alpha_{j}\right)\right| .
$$


Now tending $n$ to infinity in the last inequality, and using the assumptions of Lemma 14, we obtain that

$$
\lim _{n \rightarrow+\infty}\left|\phi_{n}\left(\alpha_{1}, \ldots, \alpha_{N}\right)-\prod_{j=1}^{N} \tilde{\phi}_{j}\left(\alpha_{j}\right)\right|=0
$$

which completes the proof of Lemma 14.

\subsection{A tool for the tightness property under association}

Proposition 5. Let $\left(X_{i, n}, i \in \mathbb{N}, n \in \mathbb{N}\right)$ be an array of centered, stationary and associated real valued random variables with finite second moment. Recall that $V_{n}(1 / \delta)=\frac{1}{\delta} \operatorname{Var} S_{n}(\delta)$. For any positive numbers $\epsilon$, a there exists a positive constant $K$ depending only on $\epsilon$ and a such that

$\frac{1}{\delta} \mathbb{P}\left(\sup _{0 \leq t \leq s \leq \delta}\left|S_{n}(t)\right| \wedge\left|S_{n}(s)-a\right| \geq 2 \epsilon,\left|S_{n}(\delta)-a\right| \leq \epsilon\right) \leq K n \sum_{r=1}^{n} \operatorname{Cov}\left(X_{0, n}, X_{r, n}\right)+K \sqrt{\delta} n \mathbb{E}\left(X_{0, n}^{2}\right) \sqrt{V_{n}(1 / \delta)}$

Proof of Proposition 5. Let $f_{a}$ and $g_{a}$ be defined as in Lemma 5 in [8]. The function $g_{a}$ is nonnegative, two times continuously differentiable, $g_{a}(x)=1$ if $|x-a| \leq \epsilon$ and $g_{a}(x)=0$ if $|x-a| \geq 3 \epsilon / 2$. The function $f_{a}(x, y)$ is defined by $f_{a}(x, y)=h_{\epsilon}(x) k_{\epsilon}(y)$, where $h_{\epsilon}$ and $k_{\epsilon}$ are nonnegative, bounded by 1 , lipchitz functions fulfilling

$$
\begin{aligned}
& h_{\epsilon}(x)=1, \text { if }|x| \geq 2 \epsilon \quad \text { and } h_{\epsilon}(x)=0 \text { if }|x| \leq \epsilon \\
& k_{\epsilon}(y)=1, \text { if }|y-a| \geq 2 \epsilon \quad \text { and } k_{\epsilon}(y)=0 \text { if }|y-a| \leq \frac{3 \epsilon}{2}
\end{aligned}
$$

Note that we always have $f_{a}(x, y) g_{a}(y)=0$. Clearly

$$
\frac{1}{\delta} \mathbb{P}\left(\sup _{0 \leq t \leq s \leq \delta}\left|S_{n}(t)\right| \wedge\left|S_{n}(s)-a\right| \geq 2 \epsilon,\left|S_{n}(\delta)-a\right| \leq \epsilon\right) \leq \frac{1}{\delta} \mathbb{E}\left(f^{*}([n \delta]) g_{a}\left(S_{[n \delta], n}\right)\right),
$$

where $f^{*}(k)=\max \left\{f_{a}\left(S_{i, n}, S_{j, n}\right), 1 \leq i \leq j \leq k\right\}$. In order to evaluate the right hand side of the last inequality, we start from the equality (3.44) in [8].

$$
\begin{aligned}
f^{*}([n \delta]) g_{a}\left(S_{[n \delta], n}\right)= & \sum_{k=1}^{[n \delta]} f^{*}(k-1)\left[g_{a}\left(S_{k, n}\right)-g_{a}\left(S_{k-1, n}\right)-X_{k, n} g_{a}^{\prime}\left(S_{k-1, n}\right)\right] \\
& +\sum_{k=1}^{[n \delta]} X_{k, n} f^{*}(k-1) g_{a}^{\prime}\left(S_{k-1, n}\right)=I_{n, \delta}+I I_{n, \delta} .
\end{aligned}
$$

Control of $\mathbb{E}\left(I_{n, \delta}\right)$. Let $X_{k, n}^{*}$ be a random variable having the same law as $X_{k, n}$ and independent of the sequence $\left(X_{k, n}\right)_{k, n}$. Define $H_{k-1, n}(x)=g_{a}\left(S_{k-1, n}+x\right)-g_{a}\left(S_{k-1, n}\right)-x g_{a}^{\prime}\left(S_{k-1, n}\right)$. Clearly

$$
f^{*}(k-1) H_{k-1, n}\left(X_{k, n}\right)=f^{*}(k-1)\left(H_{k-1, n}\left(X_{k, n}\right)-H_{k-1, n}\left(X_{k, n}^{*}\right)\right)+f^{*}(k-1) H_{k-1, n}\left(X_{k, n}^{*}\right) .
$$

Let us control the first term on the right hand of the last inequality. The function $x \rightarrow H_{k-1, n}(x)$ is one time continuously differentiable, hence

$$
H_{k-1, n}\left(X_{k, n}\right)-H_{k-1, n}\left(X_{k, n}^{*}\right)=\int\left(\mathbb{1}_{x \leq X_{k, n}}-\mathbb{1}_{x \leq X_{k, n}^{*}}\right) H_{k-1, n}^{\prime}(x) \mathrm{d} x
$$


Since $X_{k, n}$ and $X_{k, n}^{*}$ have the same distribution, we infer that

$$
\mathbb{E}\left(f^{*}(k-1)\left(H_{k-1, n}\left(X_{k, n}\right)-H_{k-1, n}\left(X_{k, n}^{*}\right)\right)\right)=\int \operatorname{Cov}\left(\mathbb{1}_{x \leq X_{k, n}}-\mathbb{1}_{x \leq X_{k, n}^{*}}, H_{k-1, n}^{\prime}(x) f^{*}(k-1)\right) \mathrm{d} x .
$$

Define $h\left(X_{1, n}, \ldots, X_{k-1, n}\right)=H_{k-1, n}^{\prime}(x) f^{*}(k-1)=\left[g_{a}^{\prime}\left(S_{k-1, n}+x\right)-g_{a}^{\prime}\left(S_{k-1, n}\right)\right] f^{*}(k-1)$. It is not hard to check that the function $h$ is coordinatewise $K$ lipshitz, for some $K$ depending only on $a$ and $\epsilon$. This fact together with the association property of the vector $\left(\mathbb{1}_{x \leq X_{k, n}}, X_{1, n}, \ldots, X_{k-1, n}\right)$ ensures that

$$
\left|\operatorname{Cov}\left(\mathbb{1}_{x \leq X_{k, n}}, H_{k-1, n}^{\prime}(x) f^{*}(k-1)\right)\right| \leq K \operatorname{Cov}\left(\mathbb{1}_{x \leq X_{k, n}}, S_{k-1, n}\right) .
$$

The last inequality together with (4.30), Fubini's lemma and the fact that

$$
X_{k, n}-X_{k, n}^{*}=\int\left(\mathbb{1}_{x \leq X_{k, n}}-\mathbb{1}_{x \leq X_{k, n}^{*}}\right) \mathrm{d} x
$$

leads to

$$
\left|\mathbb{E}\left(f^{*}(k-1)\left[H_{k-1, n}\left(X_{k, n}\right)-H_{k-1, n}\left(X_{k, n}^{*}\right)\right]\right)\right| \leq K \operatorname{Cov}\left(X_{k, n}, S_{k-1, n}\right) .
$$

In order to control $\mathbb{E}\left(f^{*}(k-1) H_{k-1, n}\left(X_{k, n}^{*}\right)\right)$, we note that $\left|H_{k-1, n}(x)\right| \leq\left\|g_{a}^{\prime \prime}\right\|_{\infty} x^{2}$ and we use the independence between $X_{k, n}^{*}$ and the sequence $\left(X_{k, n}\right)_{k, n}$. We obtain that

$$
\left|\mathbb{E}\left(f^{*}(k-1) H_{k-1, n}\left(X_{k, n}^{*}\right)\right)\right| \leq\left\|g_{a}^{\prime \prime}\right\|_{\infty} \mathbb{E}\left(f^{*}(k-1)\right) \mathbb{E}\left(X_{k, n}^{* 2}\right) .
$$

Bearing in mind the definition of $f_{a}$ and applying Lemma 8 we obtain that

$$
\mathbb{E}\left(f^{*}(k-1)\right) \leq C_{\epsilon} \mathbb{E}\left(S_{k-1, n}^{*}\right) \leq \sqrt{2} C_{\epsilon}\left(\operatorname{Var} S_{k-1, n}\right)^{1 / 2},
$$

where $C_{\epsilon}$ denotes some positive constant depending only on $\epsilon$ which may be different from line to line. Consequently

$$
\left|\mathbb{E}\left(f^{*}(k-1) H_{k-1, n}\left(X_{k, n}^{*}\right)\right)\right| \leq C_{\epsilon}\left\|g_{a}^{\prime \prime}\right\|_{\infty}\left(\operatorname{Var} S_{k-1, n}\right)^{1 / 2} \mathbb{E}\left(X_{k, n}^{2}\right) .
$$

From (4.31), (4.32) and (4.29), we infer that

$$
\begin{aligned}
\left|\mathbb{E}\left(I_{n, \delta}\right)\right| & \leq K \sum_{k=1}^{[n \delta]} \operatorname{Cov}\left(X_{k, n}, S_{k-1, n}\right)+\left\|g_{a}^{\prime \prime}\right\|_{\infty} C_{\epsilon} \sum_{k=1}^{[n \delta]}\left(\operatorname{Var} S_{k-1, n}\right)^{1 / 2} \mathbb{E}\left(X_{k, n}^{2}\right) \\
& \leq K \delta n \sum_{r=1}^{n} \operatorname{Cov}\left(X_{0, n}, X_{r, n}\right)+\left\|g_{a}^{\prime \prime}\right\|_{\infty} C_{\epsilon} n \delta \mathbb{E}\left(X_{0, n}^{2}\right)\left(\operatorname{Var} S_{[n \delta], n}\right)^{1 / 2}
\end{aligned}
$$

The last inequality holds since the sequence $\left(\operatorname{Var} S_{k, n}\right.$ ) is nondecreasing in $k$, for each fixed $n$ (this is due to the association).

Control of $\mathbb{E}\left(I I_{n, \delta}\right)$. Define the function $h$ by $h\left(X_{1, n}, \ldots, X_{k-1, n}\right)=f^{*}(k-1) g_{a}^{\prime}\left(S_{k-1, n}\right)$. It is easy to see that this function is coordinatewise $K$-lipshitz, for some $K$ depending only on $a$ and $\epsilon$. Lemma 9 implies that $\left|\operatorname{Cov}\left(X_{k, n}, f^{*}(k-1) g_{a}^{\prime}\left(S_{k-1, n}\right)\right)\right| \leq K \operatorname{Cov}\left(X_{k, n}, S_{k-1, n}\right)$. We infer that

$$
\left|\mathbb{E}\left(I I_{n, \delta}\right)\right| \leq K \sum_{k=1}^{[n \delta]} \operatorname{Cov}\left(X_{k, n}, S_{k-1, n}\right) .
$$

Proposition 5 follows from (4.27), (4.28), (4.33) and (4.34). 
We have now all the ingredients for the proofs of Theorems 2 and 3.

\subsection{Proof of Theorem 2}

Proof of (2.7). The existence of the distribution function $G$ as described by (2.7) is a consequence of Lemma 13.

Proof of the convergence in distribution of $S_{n}(t)$ for $t \in[0,1]$. It suffices to prove this statement for $t=1$. We shall use Theorem 1, Proposition 1 and Lemma 13. Condition (1.1) follows from (2.2) and (2.3) (cf. Lem. 11). Conditions $\left(\mathcal{C}_{1}\right)$ and $\left(\mathcal{C}_{2}\right)$ are satisfied using respectively Lemmas 12 and 10 . Condition $\left(\mathcal{C}_{3}\right)$ follows from Lemma 13 , since by assumption the function $G$ is continuous at 0 .

Hence $(1.1),\left(\mathcal{C}_{1}\right),\left(\mathcal{C}_{2}\right)$ and $\left(\mathcal{C}_{3}\right)$ are satisfied under the assumptions of Theorem 2 (i.e. under $(2.2),(2.3)$, B'(N), (2.5), (2.6) and the continuity of $G$ at 0.$)$ Theorem 1 and Proposition 1 apply: $S_{n}(1)$ converges in distribution to the probability $\mu_{F}^{1}$, if and only if $F$ fulfills (1.8). In view of Lemmas 13 and 10, Condition (1.8) holds for

$$
F(x)=G(x)+\mathbb{1}_{0 \leq x}(F(\infty)-G(\infty)) .
$$

This implies that $S_{n}(1)$ converges in distribution to the probability $\mu_{F}^{1}$, where $F$ is defined by (4.35).

Proof of the convergence of the finite dimensional distributions. This proof is a consequence of the following lemma

Lemma 15. Let $\left(X_{i, n}\right)_{i \in \mathbb{N}, n \in \mathbb{N}}$ be an array of centered, stationary and associated real valued random variables such that $\mathbb{E}\left(X_{0, n}^{2}\right)$ tends to 0 as $n$ goes to infinity. Suppose that Conditions (2.2) and (2.3) hold. If moreover $S_{n}(t)$ converges in distribution to the probability measure $\mu_{F}^{t}$ for any $t \in[0,1]$, then the finite dimensional distributions of the process $\left\{S_{n}(t), t \in[0,1]\right\}$ converge to those of the Lévy process with law $\mu_{F}$.

Proof of Lemma 15. Let $0=t_{-1} \leq t_{0} \leq \ldots \leq t_{m} \leq t_{m+1}=1$ be fixed. Our aim is to apply Lemma 14 to the associated sequence $U_{i, n}=\left(S_{n}\left(t_{i}\right)-S_{n}\left(t_{i-1}\right)\right)$ for $i \geq 0$. From the assumptions of Lemma 15 , we infer that $U_{i, n}$ converges in distribution to $\mu_{F}^{t_{i}-t_{i-1}}$ for any $0 \leq i \leq m+1$. To prove Lemma 15, it suffices then to check the limit (4.23). Define

$$
\mathcal{A}_{N}(n)=n \mathbb{E}\left(X_{0, n}^{2}\right)+2 n \sum_{r=1}^{N-1} \operatorname{Cov}\left(X_{0, n}, X_{r, n}\right) .
$$

Using the same arguments as in Lemma 10, we infer from Conditions (2.2), (2.3) and the asymptotic negligibility of $\left(X_{0, n}\right)$ that

$$
\lim _{N \rightarrow+\infty} \limsup _{n \rightarrow+\infty}\left|\operatorname{Var}\left(S_{n}\left(t_{i}\right)-S_{n}\left(t_{i-1}\right)\right)-\left(t_{i}-t_{i-1}\right) \mathcal{A}_{N}(n)\right|=0 .
$$

Since $S_{n}(1)=\sum_{i=0}^{m+1} U_{i, n}$, we have that

$$
2 \sum_{0 \leq i \neq j \leq m+1} \operatorname{Cov}\left(U_{i, n}, U_{j, n}\right)=\operatorname{Var} S_{n}(1)-\sum_{i=0}^{m+1} \operatorname{Var} U_{i, n}
$$

and consequently

$$
2 \sum_{0 \leq i \neq j \leq m+1} \operatorname{Cov}\left(U_{i, n}, U_{j, n}\right) \leq\left|\operatorname{Var} S_{n}(1)-\mathcal{A}_{N}(n)\right|+\sum_{i=0}^{m+1}\left|\operatorname{Var} U_{i, n}-\left(t_{i}-t_{i-1}\right) \mathcal{A}_{N}(n)\right|,
$$

and (4.23) follows from (4.36), (2.3) and (4.5). Applying Lemma 14, we conclude that the $m+1$-tuple $\left(S_{n}\left(t_{0}\right), S_{n}\left(t_{1}\right)-S_{n}\left(t_{0}\right), . ., S_{n}\left(t_{m}\right)-S_{n}\left(t_{m-1}\right)\right)$ converges in distribution to $\left(Y\left(t_{0}\right), \ldots, Y\left(t_{m}\right)-Y\left(t_{m-1}\right)\right)$, where the process $(Y(t))_{t \geq 0}$ has independent components and $Y\left(t_{i+1}\right)-Y\left(t_{i}\right)$ is distributed as $\mu_{F}^{t_{i+1}-t_{i}}$. 


\subsection{Proof of Theorem 3 and Corollary 2}

Convergence of the finite dimensional distributions. This follows from Theorem 2.

The tightness property. According to Lemma 5 in $[8]$ the process $\left\{S_{n}(t), t \in[0,1]\right\}$ is tight in $D([0,1])$ as soon as

$$
\lim _{\delta \rightarrow 0} \limsup _{n \rightarrow+\infty} \frac{1}{\delta} \mathbb{P}\left(\sup _{0 \leq t \leq s \leq \delta}\left|S_{n}(t)\right| \wedge\left|S_{n}(\delta)-S_{n}(s)\right| \geq 4 \epsilon\right)=0 .
$$

Let $\eta$ be a fixed positive real number. Recall that $f_{\eta}(x)=(x \wedge \eta) \vee(-\eta)$ is a nondecreasing and 1-lipshitz function and that

$$
S_{n}(s)=\sum_{i=0}^{[n s]} X_{i, n}(\eta)+\sum_{i=0}^{[n s]} X_{i, n}^{(\eta)}=S_{n, \eta}(s)+S_{n}^{(\eta)}(s)
$$

Consequently

$$
\left|S_{n}(t)\right| \wedge\left|S_{n}(\delta)-S_{n}(s)\right| \leq\left|S_{n}^{(\eta)}(t)\right| \wedge\left|S_{n}^{(\eta)}(\delta)-S_{n}^{(\eta)}(s)\right|+\left|S_{n, \eta}(t)\right| \vee\left|S_{n, \eta}(\delta)-S_{n, \eta}(s)\right|
$$

We infer that

$$
\mathbb{P}\left(\sup _{0 \leq t \leq s \leq \delta}\left|S_{n}(t)\right| \wedge\left|S_{n}(\delta)-S_{n}(s)\right| \geq 4 \epsilon\right) \leq I_{\eta, n}(\delta)+J_{\eta, n}(\delta)+K_{\eta, n}(\delta)
$$

where

$$
\begin{aligned}
I_{\eta, n}(\delta) & =\mathbb{P}\left(\sup _{0 \leq t \leq s \leq \delta}\left|S_{n}^{(\eta)}(t)\right| \wedge\left|S_{n}^{(\eta)}(\delta)-S_{n}^{(\eta)}(s)\right| \geq 3 \epsilon\right) \\
J_{\eta, n}(\delta) & =\mathbb{P}\left(\sup _{0 \leq t \leq \delta}\left|S_{n, \eta}(t)\right| \geq \epsilon\right) \\
K_{\eta, n}(\delta) & =\mathbb{P}\left(\sup _{0 \leq s \leq \delta}\left|S_{n, \eta}(\delta)-S_{n, \eta}(s)\right| \geq \epsilon\right) .
\end{aligned}
$$

Control of $I_{\eta, n}(\delta)$. The sequence $\left(X_{i, n}^{(\eta)}\right)$ is still associated (see the proof of Lemma 10). From (4.4) and (4.5) of Lemma 10, we infer that

$$
\limsup _{\delta \rightarrow 0} \limsup _{\eta \rightarrow 0} \limsup _{n \rightarrow+\infty} \frac{1}{\delta} \operatorname{Var}\left(S_{n}^{(\eta)}(\delta)\right) \leq \limsup _{\delta \rightarrow 0} \limsup _{n \rightarrow+\infty} \frac{1}{\delta} \operatorname{Var}\left(S_{n}(\delta)\right)<\infty .
$$

Hence, applying Proposition 5 to the associated sequence $\left(X_{i, n}^{(\eta)}\right)$, we obtain that

$$
\begin{aligned}
& \limsup _{\delta \rightarrow 0} \limsup _{\eta \rightarrow 0} \limsup _{n \rightarrow+\infty} \frac{1}{\delta} \mathbb{P}\left(\sup _{0 \leq t \leq s \leq \delta}\left|S_{n}^{(\eta)}(t)\right| \wedge\left|S_{n}^{(\eta)}(s)-a\right| \geq 2 \epsilon,\left|S_{n}^{(\eta)}(\delta)-a\right| \leq \epsilon\right) \\
& \leq K \limsup _{\eta \rightarrow 0} \limsup _{n \rightarrow+\infty} n \sum_{r=1}^{n} \operatorname{Cov}\left(X_{0, n}^{(\eta)}, X_{r, n}^{(\eta)}\right),
\end{aligned}
$$

provided that Conditions (2.2) and (2.3) hold. Consequently, we infer from (2.8) that

$$
\lim _{\delta \rightarrow 0} \limsup _{\eta \rightarrow 0} \limsup _{n \rightarrow+\infty} \frac{1}{\delta} I_{\eta, n}(\delta)=0 .
$$


Control of $J_{\eta, n}(\delta)$. Using the same arguments as for $\left(X_{r, n}^{(\eta)}\right)$, we infer that the sequence $\left(X_{r, n}(\eta)\right)$ is a sequence of bounded and associated random variables. We now apply $(4.2)$ to the sequence $\left(X_{r, n}(\eta)\right)$ with $\lambda=\epsilon\left(\operatorname{Var} S_{n, \eta}(\delta)\right)^{-1 / 2}$. We obtain that

$$
\mathbb{P}\left(\sup _{0 \leq t \leq \delta}\left|S_{n, \eta}(t)\right| \geq \epsilon\right) \leq 2 \mathbb{P}\left(\left|S_{n, \eta}(\delta)\right| \geq \epsilon-\sqrt{2 \operatorname{Var} S_{n, \eta}(\delta)}\right)
$$

From (4.4) and (4.5) of Lemma 10, we infer that

$$
\limsup _{n \rightarrow+\infty} \operatorname{Var}\left(S_{n, \eta}(\delta)\right) \leq \limsup _{n \rightarrow+\infty} \mathbb{E}\left(S_{n}^{2}(\delta)\right)<C \delta
$$

Hence, for $n$ large enough and $\delta$ small enough, we have that

$$
\mathbb{P}\left(\left|S_{n, \eta}(\delta)\right| \geq \epsilon-\sqrt{2 \operatorname{Var} S_{n, \eta}(\delta)}\right) \leq \mathbb{P}\left(\left|S_{n, \eta}(\delta)\right| \geq \frac{\epsilon}{2}\right) \leq \frac{4}{\epsilon^{2}} \mathbb{E}\left(S_{n, \eta}^{2}(\delta) \mathbb{I}_{2\left|S_{n, \eta}(\delta)\right| \geq \epsilon}\right) .
$$

Applying Lemma 12 with $\delta=1 / p$, we obtain that

$$
\lim _{\delta \rightarrow 0} \limsup _{\eta \rightarrow 0} \limsup _{n \rightarrow+\infty} \frac{1}{\delta} \mathbb{E}\left(S_{n, \eta}^{2}(\delta) \mathbb{I}_{\left|S_{n, \eta}(\delta)\right| \geq \frac{\epsilon}{2}}\right)=0
$$

provided that Conditions (2.2) and (2.3) hold. This fact, together with (4.41) and (4.42), implies that

$$
\lim _{\delta \rightarrow 0} \limsup _{\eta \rightarrow 0} \limsup _{n \rightarrow+\infty} \frac{1}{\delta} J_{\eta, n}(\delta)=0 .
$$

Control of $K_{\eta, n}(\delta)$. Define $\tilde{S}_{k, \eta}=\sum_{i=0}^{k} Y_{i, n}(\eta)$, with $Y_{i, n}(\eta)=X_{[n \delta]-i, n}(\eta)$. We have that

$$
\mathbb{P}\left(\sup _{0 \leq s \leq \delta}\left|S_{n, \eta}(\delta)-S_{n, \eta}(s)\right| \geq \epsilon\right)=\mathbb{P}\left(\max _{0 \leq k \leq[n \delta]-1}\left|\tilde{S}_{k, \eta}\right| \geq \epsilon\right)
$$

Noting that $\tilde{S}_{[n \delta]-1, \eta}=S_{n, \eta}(\delta)$, we conclude that the term $K_{\eta, n}(\delta)$ is controlled exactly as $J_{\eta, n}(\delta)$. Hence we get under Conditions (2.2) and (2.3) :

$$
\lim _{\delta \rightarrow 0} \limsup _{\eta \rightarrow 0} \limsup _{n \rightarrow+\infty} \frac{1}{\delta} K_{\eta, n}(\delta)=0
$$

Collecting (4.38), (4.40), (4.43) and (4.44), we obtain (4.37) and Theorem 3 follows.

Proof of Corollary 2. From the first remark following Theorem 3, we infer that the assumptions of Corollary 2 imply the tightness of $\left\{S_{n}(t), t \in[0,1]\right\}$. The weak convergence of the finite dimensional distributions follows from Corollary 1, Newman's inequality and Lemma 15. As it was noticed before, in the case $N_{a, 0}(X)=1$, the sequence $\left(S_{n}(1)\right)$ behaves as the sum of i.i.d. random variables distributed as $X_{0, n}$. In fact, arguing as in the proof of Lemma 11, we infer that

$$
\mid \mathbb{E} \exp \left(i z S_{n}(t)-\left(\mathbb{E}\left(\exp \left(i z S_{m, n}\right)\right)\right)^{[[n t] / m]}|\leq m| z|\mathbb{E}| X_{0, n} \mid+\frac{z^{2}}{2} n \sum_{r=1}^{n} \operatorname{Cov}\left(X_{0, n}, X_{r, n}\right)+\frac{z^{2} m}{2} \operatorname{Var}\left(X_{0, n}\right) .\right.
$$

Consequently, if $N_{a, 0}(X)=1$ then $\mathcal{E}=\mathbb{N}^{*}$. 


\subsection{Proofs of Corollaries 3 and 4}

Proof of Corollary 3. In view of Proposition 3, it suffices to check Conditions (2.8) and (2.11) with $\lambda=0$. We deduce from (4.6) and some elementary estimations that, for any $N>0$,

$$
n \sum_{r=1}^{n} \operatorname{Cov}\left(X_{0, n}^{(\eta)}, X_{r, n}^{(\eta)}\right) \leq n N \mathbb{E}\left(X_{0, n}^{2} \mathbb{I}_{\left|X_{0, n}\right| \geq \eta}\right)+n \sum_{r=N}^{n} \operatorname{Cov}\left(X_{0, n}, X_{r, n}\right) .
$$

Hence, we infer from (2.2) and (2.13) that, for any $\eta>0$,

$$
\lim _{n \rightarrow+\infty} n \sum_{r=1}^{n} \operatorname{Cov}\left(X_{0, n}^{(\eta)}, X_{r, n}^{(\eta)}\right)=0
$$

so that (2.8) holds. Clearly $\mathbb{E}\left(X_{0, n}^{(\eta) 2}\right) \leq \mathbb{E}\left(X_{0, n}^{2} \mathbb{I}_{\left|X_{0, n}\right| \geq \eta}\right)$, and Lindeberg's Condition (2.13) implies that $\lim _{n \rightarrow+\infty} n \mathbb{E}\left(X_{0, n}^{(\eta) 2}\right)=0$ for any positive $\eta$. Consequently, (2.11) holds with $\lambda=0$ and Corollary 3 follows.

Proof of Corollary 4. We first prove that, if (2.14) holds, then

$$
\lim _{\eta \rightarrow 0} \limsup _{n \rightarrow+\infty} n \mathbb{E}\left(X_{0, n}^{2}(\eta)\right)=0
$$

Clearly

$$
n \mathbb{E}\left(X_{0, n}^{2}(\eta)\right) \leq n \mathbb{E}\left(X_{0, n}^{2} \mathbb{I}_{\left|X_{0, n}\right| \leq \eta}\right)+\eta^{2} n \mathbb{P}\left(\left|X_{0, n}\right| \geq \eta\right) .
$$

From (2.14), the first term on right hand satisfies

$$
\lim _{\eta \rightarrow 0} \limsup _{n \rightarrow+\infty} n \mathbb{E}\left(X_{0, n}^{2} \mathbb{I}_{\left|X_{0, n}\right| \leq \eta}\right)=0
$$

Let $\epsilon$ be any positive number greater than $\eta$. Clearly

$$
\eta^{2} n \mathbb{P}\left(\left|X_{0, n}\right| \geq \eta\right) \leq \eta^{2} n \mathbb{P}\left(\left|X_{0, n}\right| \geq \epsilon\right)+\eta^{2} n \mathbb{P}\left(\eta \leq\left|X_{0, n}\right| \leq \epsilon\right) \leq n \frac{\eta^{2}}{\epsilon^{2}} \mathbb{E}\left(X_{0, n}^{2}\right)+n \mathbb{E}\left(X_{0, n}^{2} \mathbb{I}_{\left|X_{0, n}\right| \leq \epsilon}\right)
$$

This bound combined with (4.48) and the uniform boundedness of $\left(n \mathbb{E}\left(X_{0, n}^{2}\right)\right)_{n}$ imply that

$$
\lim _{\eta \rightarrow 0} \limsup _{n \rightarrow+\infty} \eta^{2} n \mathbb{P}\left(\left|X_{0, n}\right| \geq \eta\right)=0
$$

and (4.46) follows from (4.47), (4.48) and (4.49).

Proof of 1. We have

$$
\begin{aligned}
n \sum_{r=1}^{n} \operatorname{Cov}\left(X_{0, n}, X_{r, n}\right) \leq & n \sum_{r=1}^{N} \operatorname{Cov}\left(X_{0, n}^{(\eta)}, X_{r, n}^{(\eta)}\right)+3 N\left(n \mathbb{E}\left(X_{0, n}^{2}(\eta)\right)\right)^{1 / 2}\left(n \mathbb{E}\left(X_{0, n}^{2}\right)\right)^{1 / 2} \\
& +n \sum_{r=N}^{n} \operatorname{Cov}\left(X_{0, n}, X_{r, n}\right) .
\end{aligned}
$$

The last inequality proves that the limit in (2.9) can be deduced from (2.2), (2.8) and from (2.14) (which ensures (4.46)). 
Proof of 2. The proof of the second part of Corollary 4 is immediate from Corollary 2, but let us explain how it can be deduced from the unifying Proposition 3. The limit in $(2.9)$ is $(2.2)$ with $N_{a, 0}(X)=1$. Condition (2.3) follows from (2.9) and from the second limit in (2.14) with $F(\infty)=\lambda$. Clearly (2.8) follows from (4.6) and (2.9). It remains to prove the limit in (2.11) and in (2.12) with $\sigma^{2}=0$. The limit in (4.46), together with standard estimations based on Hölder's inequality, and the uniform boundedness of $\left(n \mathbb{E}\left(X_{0, n}^{(\eta) 2}\right)\right)_{n}$ imply that

$$
\lim _{\eta \rightarrow 0} \limsup _{n \rightarrow+\infty}\left(n \mathbb{E}\left(X_{0, n}^{2}\right)-n \mathbb{E}\left(X_{0, n}^{(\eta) 2}\right)\right)=0
$$

Combining (4.50) and (2.9), we obtain (2.12) with $\sigma^{2}=0$.

Let us now check (2.11). Clearly

$$
n \mathbb{E}\left(X_{0, n}^{(\eta) 2}\left(1 \wedge\left|X_{0, n}^{(\eta)}-1\right|\right)\right) \leq 2 n \mathbb{E}\left(X_{0, n}^{2}\left(1 \wedge\left|X_{0, n}-1\right|\right)\right)+2 n \mathbb{E}\left(X_{0, n}^{2}(\eta)\right)+2 \eta n \mathbb{E}\left(X_{0, n}^{2}\right) .
$$

This bound together with (2.14) and (4.46) implies that

$$
\lim _{\eta \rightarrow 0} \limsup _{n \rightarrow+\infty} n \mathbb{E}\left(X_{0, n}^{(\eta) 2}\left(1 \wedge\left|X_{0, n}^{(\eta)}-1\right|\right)\right)=0 .
$$

Hence (2.11) follows from (4.50), (4.51) and from the second limit in (2.14).

\section{On the Rate of Poisson Convergence}

To prove Proposition 4, we shall use the following result, which applies to random variables satisfying only $(4.22)$

Theorem 4. Let $\left(X_{i, n}\right)_{i \in \mathbb{N}, n \in \mathbb{N}}$ be an array of stationary and centered random variables, such that $\mathbb{E}\left(X_{0, n}^{2}\right)$ tends to 0 as $n$ goes to infinity. Suppose that this triangular array satisfies the inequality (4.22) and that it is uniformly bounded, say $\left|X_{1, n}\right| \leq 1$. For any $\lambda>0$ and $a \in \mathbb{R}$ denote by $\mu_{a, \lambda}$ the probability measure with characteristic function obtained from (1.3) by taking $F=\lambda \mathbb{I}_{[a, \infty[}$. Then

$$
\begin{aligned}
\sup _{h \in B_{1}^{3}(\mathbb{R})} \mid \mathbb{E}\left(h\left(S_{n}(1)\right)-\mu_{a, \lambda}(h)\left|\leq n \mathbb{E}\left(X_{0, n}^{2}\left(1 \wedge\left|X_{0, n}-a\right|\right)\right)+n\right| \mathbb{E}\left(X_{0, n}^{2}\right)-\frac{\lambda}{n} \mid\right. & \\
& +2 \sum_{i=1}^{n} \sum_{j=1}^{i-1}\left|\operatorname{Cov}\left(X_{i, n}, X_{j, n}\right)\right|+n \mathbb{E}\left(\epsilon_{0, n}^{2}\left(1 \wedge\left|\epsilon_{0, n}-a\right|\right)\right),
\end{aligned}
$$

where $\epsilon_{0, n}$ is a random variable with distribution $\mu_{a, \lambda / n}$.

Proof of Proposition 4. We apply Theorem 4 to the centered sequence $X_{i, n}=\mathbb{I}_{X_{i}>z_{n}}-p_{n}$ with $a=1$ and $\lambda_{n}=n p_{n}$. This sequence satisfies the inequality (4.22) as soon as the original sequence $\left(X_{i}\right)_{i}$ is associated (or negatively associated), since those properties as preserved under nondecreasing transformations $($ see $[10,18]$ respectively). Clearly

$$
\begin{aligned}
& n\left|\mathbb{E}\left(X_{0, n}^{2}\right)-\frac{\lambda_{n}}{n}\right|=n p_{n}^{2} \\
& n \mathbb{E}\left(X_{0, n}^{2}\left(1 \wedge\left|X_{0, n}-1\right|\right)\right)=n\left(1-p_{n}\right)^{2} p_{n}^{2}+n p_{n}^{2}\left(1-p_{n}\right) \leq 2 n p_{n}^{2} .
\end{aligned}
$$


It remains to control $n \mathbb{E}\left(\epsilon_{0, n}^{2}\left(1 \wedge\left|\epsilon_{0, n}-1\right|\right)\right)$, when $\epsilon_{0, n}$ has distribution $\mu_{1, p_{n}}$. We have that

$$
\begin{aligned}
n \mathbb{E}\left(\epsilon_{0, n}^{2}\left(1 \wedge\left|\epsilon_{0, n}-1\right|\right)\right) & =n p_{n}^{2} \mathrm{e}^{-p_{n}}\left(1+\left(1-p_{n}\right)^{2}+\frac{\left(2-p_{n}\right)^{2}\left(1-p_{n}\right)}{2}+\sum_{k=3}^{\infty} \frac{\left(k-p_{n}\right)^{2}}{k !} p_{n}^{k-2}\right) \\
& \leq 6 n p_{n}^{2} .
\end{aligned}
$$

Proposition 4 is proved by collecting Inequalities (5.1), (5.2), (5.3) and (5.4).

Proof of Theorem 4. We keep the same notations as in the proof of Proposition 7 in [8], with the choice $p=n$ in Notations 1 and 2 of this paper. In particular, if $f$ is any function from $\mathbb{R}$ to $\mathbb{R}$ the functions $f_{i-1, i+1}$ have been defined in Notations 2 . We write $f_{i-1, i+1}$ for $f_{i-1, i+1}(0)$.

Taking $p=n$ in the equality (3.6) in [8], we obtain that

$$
\begin{aligned}
\left|\mathbb{E}\left(h\left(V_{n, n}\right)-h\left(\Gamma_{1}\right)\right)\right| \leq & \left|\sum_{i=1}^{n} \mathbb{E}\left(X_{i, n} h_{i-1, i+1}^{\prime}\right)\right|+\left|\sum_{i=1}^{n} \mathbb{E}\left(\left(X_{i, n}^{2}-\epsilon_{i, n}^{2}\right) g_{i}(a)\right)\right| \\
& +n \mathbb{E}\left(X_{0, n}^{2}\left(1 \wedge\left|X_{0, n}-a\right|\right)\right)+n \mathbb{E}\left(\epsilon_{0, n}^{2}\left(1 \wedge\left|\epsilon_{0, n}-a\right|\right)\right),
\end{aligned}
$$

where $g_{i}(x)=\int_{0}^{1}(1-t) h_{i-1, i+1}^{\prime \prime}(t x) \mathrm{d} t$. Define

$$
D_{1, n}=\left|\sum_{i=1}^{n} \mathbb{E}\left(X_{i, n} h_{i-1, i+1}^{\prime}\right)\right| \quad \text { and } \quad D_{2, n}=\left|\sum_{i=1}^{n} \mathbb{E}\left(\left(X_{i, n}^{2}-\epsilon_{i, n}^{2}\right) g_{i}(a)\right)\right| .
$$

Control of $D_{1, n}$. The triangular array $\left(X_{i, n}\right)$ is independent of $\left(\epsilon_{i, n}\right)$ and $\left\|h^{\prime \prime}\right\|_{\infty} \leq 1$. Applying the inequality (4.22) we obtain that

$$
D_{1, n} \leq \sum_{i=1}^{n}\left|\operatorname{Cov}\left(X_{i, n}, h_{i-1, i+1}^{\prime}\right)\right| \leq \sum_{i=1}^{n} \sum_{j=1}^{i-1}\left|\operatorname{Cov}\left(X_{i, n}, X_{j, n}\right)\right| .
$$

Control of $D_{2, n}$. Since $n \mathbb{E}\left(\epsilon_{i, n}^{2}\right)=\lambda$ and $\operatorname{Cov}\left(\epsilon_{i, n}^{2}, g_{i}(a)\right)=0$, we infer that

$$
D_{2, n} \leq\left|\sum_{i=1}^{n} \operatorname{Cov}\left(X_{i, n}^{2}, g_{i}(a)\right)\right|+\left|n \mathbb{E}\left(X_{0, n}^{2}\right)-\lambda\right| .
$$

Since $\left|X_{i, n}\right| \leq 1, X_{i, n}^{2}$ may be seen as a 2-lipshitz function of $X_{i, n}$. Since $\left\|h^{\prime \prime \prime}\right\| \leq 1$, we infer that $g_{i}$ is an $1 / 2$-lipshitz function of $X_{k, n}$ for $1 \leq k<i$. Applying the inequality (4.22) we obtain that

$$
\left|\sum_{i=1}^{n} \operatorname{Cov}\left(X_{i, n}^{2}, g_{i}(a)\right)\right| \leq \sum_{i=1}^{n} \sum_{j=1}^{i-1}\left|\operatorname{Cov}\left(X_{i, n}, X_{j, n}\right)\right| .
$$

Finally,

$$
D_{2, n} \leq \sum_{i=1}^{n} \sum_{j=1}^{i-1}\left|\operatorname{Cov}\left(X_{i, n}, X_{j, n}\right)\right|+\left|n \mathbb{E}\left(X_{0, n}^{2}\right)-\lambda\right| .
$$

Theorem 4 is proved by collecting Inequalities $(5.5),(5.6)$ and (5.7). 


\section{REFERENCES}

[1] A. Araujo and E. Giné, The central limit theorem for real and Banach space valued random variables. Wiley, New York (1980).

[2] A.D. Barbour, L. Holst and S. Janson, Poisson approximation. Clarendon Press, Oxford (1992).

[3] R.E. Barlow and F. Proschan, Statistical Theory of Reliability and Life: Probability Models. Silver Spring, MD (1981).

[4] T. Birkel, On the convergence rate in the central limit theorem for associated processes. Ann. Probab. 16 (1988) $1685-1698$.

[5] A.V. Bulinski, On the convergence rates in the CLT for positively and negatively dependent random fields, in Probability Theory and Mathematical Statistics, I.A. Ibragimov and A. Yu. Zaitsev Eds. Gordon and Breach Publishers, Singapore, (1996) 3-14.

[6] L.H.Y. Chen, Poisson approximation for dependent trials. Ann. Probab. 3 (1975) 534-545.

[7] J.T. Cox and G. Grimmett, Central limit theorems for associated random variables and the percolation models. Ann. Probab. 12 (1984) 514-528.

[8] J. Dedecker and S. Louhichi, Conditional convergence to infinitely divisible distributions with finite variance. Stochastic Proc. Appl. (To appear.)

[9] P. Doukhan and S. Louhichi, A new weak dependence condition and applications to moment inequalities. Stochastic Proc. Appl. 84 (1999) 313-342.

[10] J. Esary, F. Proschan and D. Walkup, Association of random variables with applications. Ann. Math. Statist. 38 (1967) 1466-1476.

[11] C. Fortuin, P. Kastelyn and J. Ginibre, Correlation inequalities on some ordered sets. Comm. Math. Phys. 22 (1971) 89-103.

[12] B.V. Gnedenko and A.N. Kolmogorov, Limit distributions for sums of independent random variables. Addison-Wesley Publishing Company (1954).

[13] L. Holst and S. Janson, Poisson approximation using the Stein-Chen method and coupling: number of exceedances of Gaussian random variables. Ann. Probab. 18 (1990) 713-723.

[14] T. Hsing, J. Hüsler and M.R. Leadbetter, On the Exceedance Point Process for a Stationary Sequence. Probab. Theory Related Fields 78 (1988) 97-112.

[15] W.N. Hudson, H.G. Tucker and J.A Veeh, Limit distributions of sums of m-dependent Bernoulli random variables. Probab. Theory Related Fields 82 (1989) 9-17.

[16] A. Jakubowski, Minimal conditions in p-stable limit theorems. Stochastic Proc. Appl. 44 (1993) 291-327.

[17] A. Jakubowski, Minimal conditions in p-stable limit theorems -II. Stochastic Proc. Appl. 68 (1997) 1-20.

[18] K. Joag-Dev and F. Proschan, Negative association of random variables, with applications. Ann. Statist. 11 (1982) $286-295$.

[19] O. Kallenberg, Random Measures. Akademie-Verlag, Berlin (1975).

[20] M. Kobus, Generalized Poisson Distributions as Limits of Sums for Arrays of Dependent Random Vectors. J. Multi. Analysis (1995) 199-244.

[21] M.R Leadbetter, G. Lindgren and H. Rootzén, Extremes and related properties of random sequences and processes. New York, Springer (1983).

[22] C.M. Newman, Asymptotic independence and limit theorems for positively and negatively dependent random variables, in Inequalities in Statistics and Probability, Y.L. Tong Ed. IMS Lecture Notes-Monograph Series 5 (1984) 127-140.

[23] C.M. Newman, Y. Rinott and A. Tversky, Nearest neighbors and voronoi regions in certain point processes. Adv. Appl. Prob. 15 (1983) 726-751.

[24] C.M. Newman and A.L. Wright, An invariance principle for certain dependent sequences. Ann. Probab. 9 (1981) 671-675.

[25] V.V. Petrov, Limit theorems of probability theory: sequences of independent random variables. Clarendon Press, Oxford (1995).

[26] L. Pitt, Positively Correlated Normal Variables are Associated. Ann. Probab. 10 (1982) 496-499.

[27] E. Rio, Théorie asymptotique des processus aléatoires faiblement dépendants. Collection Mathématiques \& Applications. Springer, Berlin 31 (2000).

[28] K.I. Sato, Lévy processes and infinitely divisible distributions. Cambridge studies in advanced mathematics 68 (1999).

[29] C.M. Stein, A bound for the error in the normal approximation to the distribution of a sum of dependent random variables, in Proc. Sixth Berkeley Symp. Math. Statist. Probab. Univ. California Press 3 (1971) 583-602. 